\title{
11. MIOCENE TO PLIOCENE PLANKTONIC FORAMINIFERS FROM THE NORTH AOBA BASIN, SITE 832 ${ }^{1}$
}

\author{
Russell C. B. Perembo²
}

\begin{abstract}
One hundred and sixty core samples were analyzed from Hole 832B to evaluate planktonic foraminiferal datum levels, and to zone and correlate the borehole succession. A total of 32 biostratigraphic events were recognized in the interval from Core 134-832B-59R through 134-832B-73R (702.49 through 846.4 meters below seafloor [mbsf]). These include 17 first appearance datum levels (FAD), 10 last appearance datum levels (LAD), and 5 coiling-change events in trochospiral species.

The studied succession has been subdivided into nine planktonic foraminiferal zones (viz. downsequence N.22, N.21, N.20, N.19, N.18, N.17B, N.17A-N.16, N.15, N.8). The zonal index species occur in the expected stratigraphic order for zonal correlation, but some of the zonal boundaries may be diachronous compared to other localities in the western Pacific region. The FAD of Globorotalia (Truncorotalia) truncatulinoides (d'Orbigny) at $714.10 \mathrm{mbsf}$ defines the boundary between the Zone N.22 and N.21; the boundary between Zones N.21 and N.20 at 741.73 mbsf is marked by the FAD of Globorotalia (Truncorotalia) tosaensis Takayanagi and Saito. The lower boundary of Zone N.20 is placed at $747.65 \mathrm{mbsf}$, based on the FAD of Globorotalia (Truncorotalia) crassaformis s.s. (Galloway and Wissler); the FAD of Sphaeroidinella dehiscens (Parker and Jones) at 756.61 mbsf defines the boundary between Zones N.18 and N.19. The FAD of Globorotalia (Globorotalia) tumida tumida (Brady) at 811.15 mbsf marks the boundary between Zones N.18 and N.17B. The boundary between Zones N.17B and N.17A is placed at 843.52 mbsf, based on the FAD of Pulleniatina primalis Banner and Blow.

A change in depositional conditions occurs at 846.4 mbsf just below the Zone N.17B lower boundary and is marked by the first appearance of abundant planktonic foraminifers in the region. The interval between 849.13 and 856.1 mbsf is placed in undifferentiated Zones N.17A and N.16, based on the rare occurrence of Neogloboquadrina acostaensis (Blow). The sparsely fossiliferous volcanic sandstone unit between 934.19 and $955.67 \mathrm{mbsf}$ is positioned within Zone N.15 based on the presence of Globigerina (Zeaglobigerina) nepenthes Todd and Globigerinoides (Zeaglobigerina) druryi Arkers, and absence of N. acostaensis and Globorotalia (Jenkinsella) siakensis LeRoy.

An unconformity between 955.67 and 971.80 mbsf may explain the absence of Zones N.14 through N.9. Basal Zone N.8 is recognized at 971.80 to 1008.60 mbsf by the presence of Globigerinoides sicanus De Stefani and the absence of Praeorbulina and Orbulina spp.

The age of the succession between 702.49 and 1008.6 mbsf extends from the latest Pliocene or earliest Pleistocene (Zone N.22) to the earliest middle Miocene (Zone N.8). Among the datum levels evaluated here, the following events are considered to be the most reliable for time correlation in the studied region: the FADs of $G$. (T.) truncatulinoides, $G$. (T.) tosaensis, $G$. (T.) crassaformis, S. dehiscens, G. conglobatus (Brady), G. (G.) tumida tumida, and P. primalis; and the LADs of Globorotalia (Menardella) multicamerata Cushman and Jarvis, and Dentoglobigerina altispira altispira (Cushman and Jarvis). Application of a chronometric scale to part of the succession, suggests that the interval of calcareous sediment between 702.49 and 846.4 mbsf accumulated at about $30 \mathrm{~m} / \mathrm{m} . \mathrm{y}$.
\end{abstract}

\section{INTRODUCTION}

The main aims of this study are to evaluate the datum levels of important planktonic foraminiferal species from the Miocene to Pliocene in the North Aoba Basin, and, by application of planktonic foraminiferal biostratigraphy, to constrain the age of the foraminiferal succession at Site 832. This work will contribute to one of the objectives of ODP Leg 134 Site 832: unravelling the tectonic evolution of the intra-arc basin. It will also improve the understanding of the uppermost Miocene to Pliocene planktonic foraminiferal biostratigraphic sequence in low-latitude areas of the southwest Pacific region.

The Neogene planktonic foraminiferal datum planes at mid and low latitudes in the southwest Pacific were summarized by Kennett and Srinivasan (1981b, 1984) and discussed further by Srinivasan and Sinha (1991). Their work involved evaluation of datum levels in samples recovered from Deep Sea Drilling Project (DSDP) holes and showed that the stratigraphic ranges of many species vary between different latitudinal (climatic) belts. Kennett and Srinivasan (1984) recognized three faunal provinces in the southwest Pacific region: equatorial (e.g., DSDP Site 289), subtropical (e.g., DSDP Site 208),

'Greene, H.G., Collot, J.-Y., Stokking, L.B., et al., 1994. Proc. ODP, Sci. Results, 134: College Station, TX (Ocean Drilling Program).

${ }^{2}$ Department of Geology, University of Western Australia, Nedlands 6009 Perth WA. Australia. and transitional (e.g., DSDP Site 206). The latitude of North Aoba Basin is between that of Sites 289 (Ontong Java Plateau) and 208 (Lord Howe Rise).

Site 832 , located at $14^{\circ} 47.78^{\prime} \mathrm{S}, 167^{\circ} 34.35^{\prime} \mathrm{E}$ (Fig. 1) in the North Aoba Basin, was selected for this study because of its thick and relatively undisturbed succession of calcareous and volcanic facies. Hole 832B penetrated sedimentary strata to a total depth of 1106.7 meters below seafloor (mbsf), and this study is based on the foraminiferal assemblages recovered from lithostratigraphic Units V, VI, and VII of Collot, Greene, Stokking, et al. (1992). Lithostratigraphic Unit $\mathrm{V}$ is a $163.7 \mathrm{~m}$ thick foraminiferal, nannofossil, or silty limestone with occasional layers of vitric ash; Unit VI is a $86.9 \mathrm{~m}$ thick lithified volcanic sandstone; and Unit VII is a 154.1-m-thick breccia composed of $60 \%$ lithified basaltic breccia and $40 \%$ lithified volcanic sandstone and siltstone containing neritic bioclasts, including corals, algal fragments, and larger foraminifers (i.e., Amphistegina spp., Lepidocyclina sp., Miogypsina spp.).

The uppermost Miocene and Pliocene sediment contain abundant and well-preserved planktonic foraminifers and provide an ideal faunal succession for the study of biostratigraphic events. The top of the main section studied (encompassing lithostratigraphic Unit V) was chosen at $702.49 \mathrm{mbsf}$ to coincide with an unconformity revealed by the seismic reflection data, and the bottom at $846.4 \mathrm{mbsf}$ lies just above a possible unconformity at a level where many of the important foraminiferal species disappear downsequence. Within lithostratigraphic 


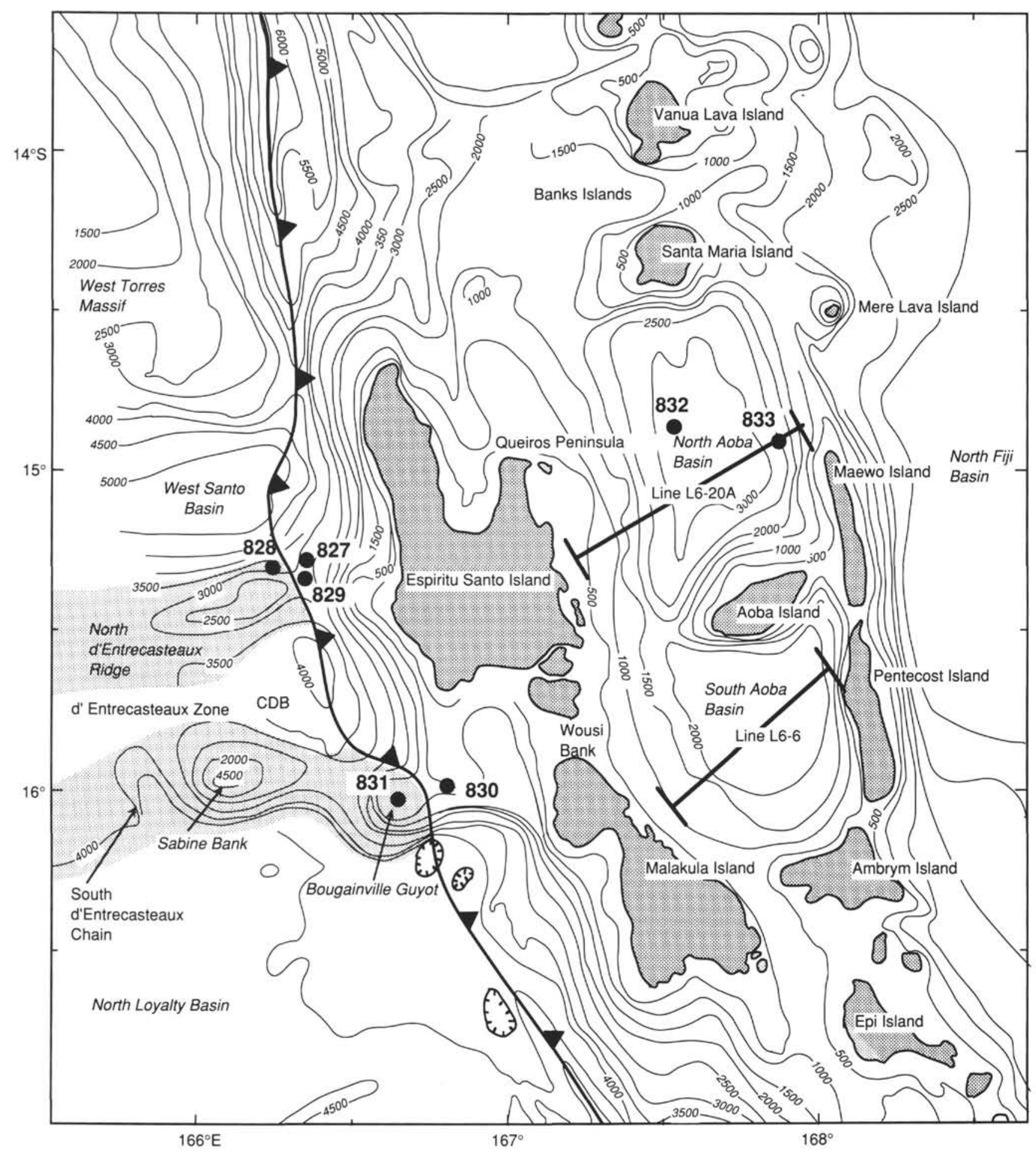

Figure 1. Location of Site 832, North Aoba Basin.

Unit V, the occurrence of abundant large planktonic foraminifers and the presence of Uvigerina hispidocostata, Cibicides wuellerstorfi, Melonis pompilioides, and Pyrgo murrhina among the benthonic species suggest that deposition occurred within the lower bathyal zone. Below $846.4 \mathrm{mbsf}$ (in lithostratigraphic Units VI and VII), few samples yielded foraminifers; these are analyzed to determine the age of the succession in this sparsely fossiliferous interval.

\section{MATERIALS AND METHODS}

This study is based on 160 friable core samples collected from Hole 134-832B. One hundred and sixteen samples yielded sufficient foraminiferal assemblages for biostratigraphic analysis. Samples were disaggregated by boiling with added detergent solution, and sieved through a $63-\mu \mathrm{m}$ screen. Washed residues from the $63-\mu \mathrm{m}$ sieve were used for 
the foraminiferal analysis. Index planktonic foraminiferal species were selectively picked and estimates of abundance were made (see explanation of Tables 1 and 2). The species were photographed using a Phillips 505 scanning electron microscope. All the specimens illustrated on Plates 1 through 6 are housed in the micropaleontological collections of the Department of Geology, University of Western Australia, Perth

\section{BIOSTRATIGRAPHIC ZONATION}

The tropical N. zonation of Blow (1969), as amended by Kennett and Srinivasan (1983), with some modifications suggested by other workers, forms the biostratigraphic framework for this study. The chronometric scale and chronostratigraphy of Berggren et al. (1985) is applied to the succession. The taxonomic concepts and species nomenclature of Kennett and Srinivasan (1983) are followed, unless otherwise stated.

Several authors have suggested some modifications to the definition of zonal boundaries. The ones important for this study are the N.22/N.21 boundary and the N.20/N.19 boundary. The boundary of Zone N.22/N.21 is defined by the first appearance of G. (T.) truncatulinoides. In a comparison of distributions within DSDP holes in the Pacific, Hills and Thierstein (1989) demonstrated that the first appearance datum of $G$. (T.) truncatulinoides was diachronous, being oldest in the southwest Pacific region. Chaproniere (1991) retained $G$. (T.) truncatulinoides as the marker species for Zone N.22/N.21boundary. $\mathrm{He}$ argued that the age of first appearance of $G$. (T.) truncatulinoides marking the zonal boundary was consistent within global low latitudes. Because the fauna of Site 832 seems similar to equatorial province assemblages of the western Pacific, the N.22/N.21 boundary based on the FAD of $G$. (T.) truncatulinoides is considered a useful level for time correlation.

Brönnimann and Resig (1971) suggested that Globorotalia acostaensis pseudopima Blow, whose first appearance Blow (1969) used to define the Zone N.20/N.19 boundary, ranged below N.19 and, therefore, Zones N.19 and N.20 could not be differentiated. Srinivasan and Kennett (1981a, their Fig. 11) separated Zone N.19-20 from Zone N.19 by the LAD of Globorotalia (Hirsutella) margaritae Bolli and Bermúdez. Heath and McGowran (1984) also recognized the boundary between Zones N.20 and N.19 at the last appearance of $G$. (H.) margaritae. However, this species has not been found at Site 832. As indicated by Haig and Perembo (1990), the first appearance of $G$. (T.) crassaformis s.s. seems to be coincident with the N.1920/N.19 boundary of Kennett and Srinivasan (1983, their Fig. 16) and this level is taken as the boundary between Zones N.20 and N.19 at Site 832.

\section{Planktonic Foraminiferal Zonation at Site $\mathbf{8 3 2}$}

Table 1 and 2 outlines the stratigraphic ranges of important planktonic species found in the studied sequence. The interval from 702.49 to 714.10 mbsf belongs within Zone N.22 based on the presence of $G$. (T.) truncatulinoides, associated with $G$. (T.) tosaensis and Globorotalia (Truncorotalia) tosaensis tenuitheca (Blow). Zone N.21 covers the interval from 714.10 to $741.73 \mathrm{mbsf}$ based on the presence of $G$. (T.) tosaensis, and the absence of $G$. (T.) truncatulinoides, and the interval from 741.73 to 747.65 mbsf belongs within Zone N.20 based on the presence of $G$. (T.) crassaformis s.s., and the absence of $G$. (T.) tosaensis. The interval from 747.65 to 756.61 mbsf encompasses Zone N.19, based on the occurrence of S. dehiscens and the absence of $G$. (T.) crassaformis s.s. Zone N.18 occurs between 756.61 to $811.15 \mathrm{mbsf}$, based on the presence of $G$. (G.) tumida tumida and the absence of $S$. dehiscens. The sediments from 811.15 to 843.52 mbsf are within Zone N.17B based on the presence of $P$. primalis, and the absence of $G$. (G.) tumida tumida. The interval between 843.52 to 856.10 mbsf includes Zone N.17A or Zone N.16, based on the occurrence of sparse assemblages including $N$. acostaensis. The vol- canic sandstone unit from 971.80 to $856.10 \mathrm{mbsf}$ belongs somewhere within Zone N.15, based on the co-occurrence of the species $G$. (Z.) nepenthes and $G$. (Z.) druryi and the absence of $N$. acostaensis and G. (J.) siakensis.

It is apparent from the foraminiferal assemblages that no indicator species from Zones N.14 through N.9 are present at Site 832. This suggests that the stratigraphic interval represented by Zones N.14 through N.9 may be missing from the Hole 832B succession. The sediments from 971.80 to 1008.60 mbsf belong to Zone N.8, as indicated by the presence of $G$. sicanus and Praeorbulina transitoria (Blow), and the absence of Orbulina spp.

\section{COMPARISON OF BIOSTRATIGRAPHIC EVENTS}

The succession of biostratigaphically important events at Site 832 is discussed below in descending stratigraphic order, and their stratigraphic distribution is charted in Figure 2. The events are numbered 1 through 32 in the text and on the figure and include first appearance datum levels (FAD), last appearance datum levels (LAD), and changes in coiling direction among trochospiral species. Full species nomenclature is given in the Figure 2 caption.

The FAD of $G$. (T.) truncatulinoides (1) at 714.10 mbsf marks the base of planktonic foraminiferal Zone N.22. Within planktonic foraminiferal Zone N.21 assemblages of $G$. (M.) menardii (2) change from sinistral to dextral coiling downsequence at $718.10 \mathrm{mbsf}$; the LAD of $G$. (M.) multicamerata (3) occurs at $718.60 \mathrm{mbsf}$; the FAD of $P$. obliquiloculata (4) and the LAD of G. fistulosus (5) at 733.00 mbsf; the FAD of $G$. (T.) crassaformis ronda (6) at 740.20 mbsf; and the FAD of $G$. (T.) tosaensis (7) and $G$. (T.) tosaensis tenuitheca (8) at 741.73 mbsf. The FAD of G. (T.) tosaensis (7) defines the base of Zone N.21.

Within Zone N.20 the LAD of Dentoglobigerina altispira altispira (9) is at $742.30 \mathrm{mbsf}$, and both the FAD of $G$. fistulosus (10) and the LAD of $P$. praecursor (11) are at 745.40 mbsf, followed by the FAD of $G$. (T.) crassaformis s.s. (12) at 747.65 mbsf, which marks the base of Zone N.20.

Events within Zone N.19 include the LAD of S. paenedehiscens (13), S. seminulina seminulina (14), S. kochi (15), and P. primalis (16) at $748.45 \mathrm{mbsf}$, and both the FAD of $S$. dehiscens (17; marking the base of the zone) and the LAD of G. (Z.) nepenthes (18) at 756.61 mbsf.

Within Zone N.18, the FAD of $P$. praecursor (19) is at 801.30 mbsf, and $N$. humerosa (20) at 805.00 mbsf. The presence of $G$. (T.) crassula (21) is sporadic throughout the interval and its FAD occurs at 809.19 mbsf together with the FAD of $G$. conglobatus (22), and the coiling changes downsequence from sinistral to dextral in $G$. (G.) tumida flexuosa (23). A change in coiling in $G$. (M.) multicamerata (24) from dextral to sinistral downsequence is noted at $809.46 \mathrm{mbsf}$, and in $P$. primalis (25) from sinistral to dextral downsequence at 810.23 mbsf. The FAD of $G$. (G.) tumida tumida (26) marking the base of Zone N.18, and the FAD of $G$. (G.) tumida flexuosa (27) occur at 811.15 mbsf.

Within Zone N.17B populations of $P$. primalis (28) change from dextral to sinistral downsequence at $815.15 \mathrm{mbsf}$; and the LAD of $N$. acostaensis (29) is noted at $816.63 \mathrm{mbsf}$. The FAD of $G$. (M.) multicamerata (30) is at $839.20 \mathrm{mbsf}$, followed by the FAD of $P$. primalis (31) at 840.93 mbsf, marking the base of Zone N.17B. The FAD of $S$. paenedehiscens (32) occurs just below this level at $846.40 \mathrm{mbsf}$.

The abundance downsequence decreases dramatically in populations of $G$. (Z.) nepenthes at $840.93 \mathrm{mbsf}$, followed by $S$. seminulina seminulina and $S$. kochi at $846.40 \mathrm{mbsf}$. Also noticeable at $846.4 \mathrm{mbsf}$ is the downhole disappearance of many of the overlying important uppermost Miocene to Pliocene foraminifers. Below 846.4 mbsf in the volcanic sandstone unit the abundance of planktonic foraminifers decreases and datum levels cannot be precisely located.

As discussed below, some differences are apparent between the events charted by Kennett and Srinivasan (1983) and the order of these events encountered in the uppermost Miocene to Pliocene suc- 
Table 1. Stratigraphic distribution of biostratigaphically important planktonic foraminiferal species from the uppermost Miocene to upper Pliocene. Relative abundance, diversity, and preservation are visually estimated, with relative abundance denoted as follows: $\mathrm{A}=\mathrm{abundant}=\mathrm{greater}$ than 10 specimens, $C=$ common $=6-10$ specimens, $F=$ few $=3-5$ specimens, and $R=$ rare $=1-2$ specimens. Preservation is indicated thusly: $G=$ good, for specimens where all morphological features are distinct identifiable, $M=$ moderate, for specimens where some features are difficult to recognize because of preservation, and $\mathbf{P}=$ poor, for specimens that are difficult to identify because of their state of preservation. Diversity in a total foraminiferal assemblage is estimated as high $(\mathrm{H})$ for greater than 5 species, and low $(\mathrm{L})$ for 1-5 species.

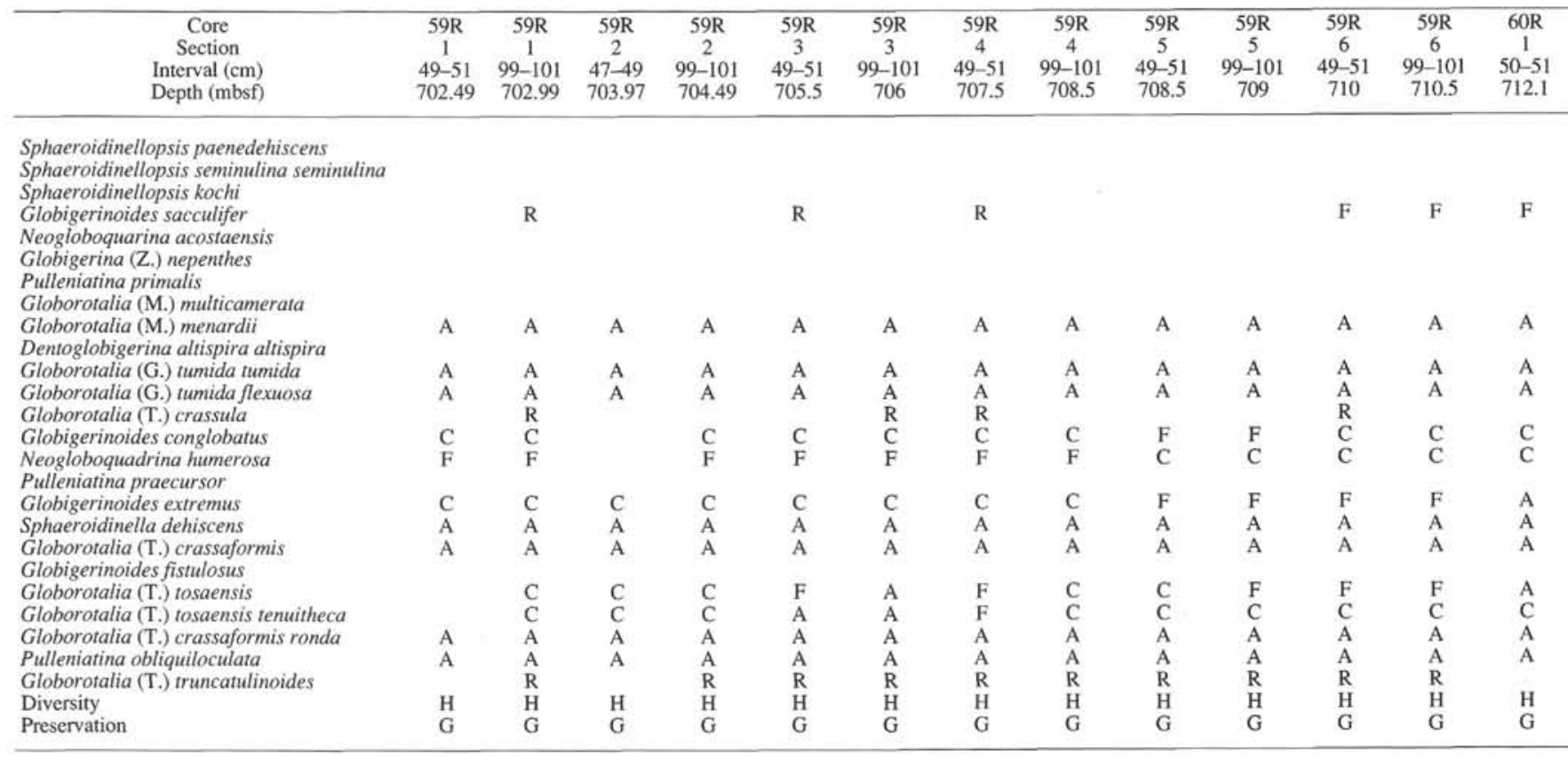

Table 1 (continued).

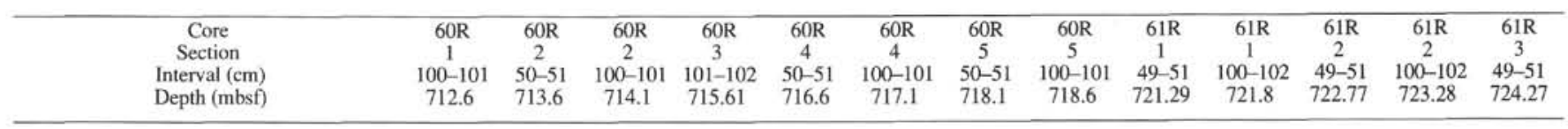

Sphaeroidinellopsis paenedehiscens

Sphaeroidinellopsis seminulina seminulina

Sphaeroidinellopsis kochi

Globigerinoides sacculifer

Neogloboquarina acostaensis

Globigerina (Z.) nepenthes

Pulleniatina primalis

Globorotalia (M.) multicamerata

Globorotalia (M.) menardii

Dentoglobigerina altispira altispira

Globorotalia (G.) tumida tumida

Globorotalia (G.) tumida flexuosa

Globorotalia (T.) crassula

Glbigerinoides conglobatus

Neogloboquadrina humerosa

Pulleniatina praecursor

Globigerinoides extremus

Sphaeroidinella dehiscens

Globorotalia (T.) crassaformis

Globigerinoides fistulosus

Globorotalia (T.) tosaensis

Globorotalia (T.) tosaensis tenuitheca

Globorotalia (T.) crassaformis ronda

Pulleniatina obliquiloculata

Globorotalia (T.) truncatulinoides

Diversity

Preservation

\begin{tabular}{|c|c|c|c|c|c|c|c|c|c|c|c|c|}
\hline A & A & C & A & $\mathrm{A}$ & C & C & $\begin{array}{l}\mathrm{C} \\
\mathrm{C}\end{array}$ & A & $\begin{array}{l}\mathrm{A} \\
\mathrm{A}\end{array}$ & $\begin{array}{l}\mathrm{A} \\
\mathrm{A}\end{array}$ & A & $\mathrm{A}$ \\
\hline${ }_{\mathrm{A}}^{\mathrm{A}}$ & $\begin{array}{l}\mathrm{A} \\
\mathrm{A}\end{array}$ & C & $\begin{array}{l}\mathrm{A} \\
\mathrm{A}\end{array}$ & $\begin{array}{l}\mathrm{A} \\
\mathrm{A}\end{array}$ & $\begin{array}{l}\mathrm{C} \\
\mathrm{C}\end{array}$ & $\begin{array}{l}\mathrm{A} \\
\mathrm{A}\end{array}$ & $\stackrel{\mathrm{A}}{\mathrm{A}}$ & $\stackrel{\mathrm{A}}{\mathrm{A}}$ & $\stackrel{\mathrm{A}}{\mathrm{A}}$ & $\begin{array}{l}\mathrm{A} \\
\mathrm{A}\end{array}$ & $\begin{array}{l}\mathrm{A} \\
\mathrm{A}\end{array}$ & $\begin{array}{l}\mathrm{A} \\
\mathrm{A}\end{array}$ \\
\hline $\begin{array}{l}\mathrm{F} \\
\mathrm{F}\end{array}$ & $\begin{array}{l}F \\
F\end{array}$ & $\begin{array}{l}F \\
F\end{array}$ & $\begin{array}{l}F \\
F\end{array}$ & $\underset{F}{F}$ & $F$ & $\begin{array}{l}\mathrm{C} \\
\mathrm{C}\end{array}$ & $\begin{array}{l}\mathrm{A} \\
\mathrm{F}\end{array}$ & $\begin{array}{l}F \\
R\end{array}$ & $\stackrel{\mathrm{F}}{\mathrm{C}}$ & $\begin{array}{l}\mathrm{C} \\
\mathrm{C}\end{array}$ & $\begin{array}{l}F \\
F\end{array}$ & $\begin{array}{l}\mathrm{C} \\
\mathrm{C}\end{array}$ \\
\hline $\begin{array}{l}\text { C } \\
\text { A } \\
\text { C }\end{array}$ & $\begin{array}{l}\mathrm{A} \\
\mathrm{A} \\
\mathrm{A}\end{array}$ & $\begin{array}{l}\text { C } \\
\text { A } \\
\text { A }\end{array}$ & $\begin{array}{l}\mathrm{F} \\
\mathrm{A} \\
\mathrm{A}\end{array}$ & $\begin{array}{l}\text { C } \\
\text { C } \\
\text { A }\end{array}$ & $\begin{array}{l}\mathrm{F} \\
\mathrm{A} \\
\mathrm{F}\end{array}$ & ${ }_{\mathrm{C}}^{\mathrm{A}}$ & $\stackrel{\mathrm{A}}{\mathrm{A}}$ & $\begin{array}{l}\mathrm{C} \\
\mathrm{A} \\
\mathrm{C}\end{array}$ & $\begin{array}{l}\text { C } \\
\text { A } \\
\text { A }\end{array}$ & $\begin{array}{l}\text { C } \\
\text { A } \\
\text { C }\end{array}$ & $\begin{array}{l}\text { C } \\
\text { A } \\
\text { C }\end{array}$ & $\begin{array}{c}\mathrm{F} \\
\mathrm{A} \\
\mathrm{A}\end{array}$ \\
\hline $\begin{array}{l}\mathrm{C} \\
\mathrm{F} \\
\mathrm{A} \\
\mathrm{A} \\
\mathrm{R}\end{array}$ & $\begin{array}{l}\mathrm{C} \\
\mathrm{F} \\
\mathrm{C} \\
\mathrm{C} \\
\mathrm{R}\end{array}$ & $\begin{array}{l}\text { A } \\
\text { C } \\
\text { C } \\
\text { C } \\
\text { R }\end{array}$ & $\begin{array}{l}\mathrm{C} \\
\mathrm{A} \\
\mathrm{A} \\
\mathrm{F}\end{array}$ & $\begin{array}{l}\text { C } \\
\text { A } \\
\text { A } \\
\text { C }\end{array}$ & $\mathrm{F}$ & $\begin{array}{l}\text { C } \\
\text { C } \\
\text { C } \\
\text { F }\end{array}$ & $\begin{array}{l}\text { C } \\
\text { A } \\
\text { A } \\
\text { F }\end{array}$ & $\begin{array}{l}\text { C } \\
\text { C } \\
\text { A } \\
\text { F }\end{array}$ & $\begin{array}{l}\text { A } \\
\text { C } \\
\text { A } \\
\text { A }\end{array}$ & $\begin{array}{l}\mathrm{F} \\
\mathrm{F} \\
\mathrm{C} \\
\mathrm{A}\end{array}$ & $\begin{array}{l}\mathrm{F} \\
\mathrm{F} \\
\mathrm{C} \\
\mathrm{C}\end{array}$ & $\begin{array}{l}\mathrm{F} \\
\mathrm{R} \\
\mathrm{A} \\
\mathrm{C}\end{array}$ \\
\hline $\begin{array}{l}\mathrm{H} \\
\mathrm{G}\end{array}$ & $\underset{\mathrm{G}}{\mathrm{H}}$ & $\begin{array}{l}\mathrm{H} \\
\mathrm{G}\end{array}$ & $\begin{array}{l}\mathrm{H} \\
\mathrm{G}\end{array}$ & $\begin{array}{l}\mathrm{H} \\
\mathrm{G}\end{array}$ & $\begin{array}{l}\mathrm{H} \\
\mathrm{G}\end{array}$ & $\underset{G}{\mathrm{H}}$ & $\begin{array}{l}\mathrm{G} \\
\text { th }\end{array}$ & $\underset{\mathrm{G}}{\mathrm{H}}$ & $\begin{array}{l}\mathrm{H} \\
\mathrm{G}\end{array}$ & $\begin{array}{l}\mathrm{H} \\
\mathrm{G}\end{array}$ & $\begin{array}{l}\mathrm{H} \\
\mathrm{G}\end{array}$ & $\begin{array}{l}\mathrm{G} \\
\mathrm{H}\end{array}$ \\
\hline
\end{tabular}

cession in Hole 832B. The datum levels are also compared with the succession outlined by Berggren et al. (1985) and the datum levels previously recorded at western Pacific localities close to Site 832 .

\section{First Appearance Datum Levels}

Compared to stratigraphic ranges recorded by Kennett and Srinivasan (1983), the following species have anomalous first appearances in Hole 832B: P. obliquiloculata (in Zone N.21 rather than in Zone N.20), G. fistulosus (in Zone N.20 rather than in Zone N.21), $P$. praecursor (in Zone N.18 rather than in Zone N.19), and G. (G.) tumida flexuosa (in Zone N.18 rather than in Zone N.19).

Kennett and Srinivasan (1983, their Figure 22) placed the FAD of P. obliquiloculata at the base of their Zone N.19-20 (stated in their text as Zone N.19), whereas Kennett (1973) recorded the corresponding FAD from DSDP Site 203 (Tonga Ridge) near the middle part of 
Table 1 (continued).

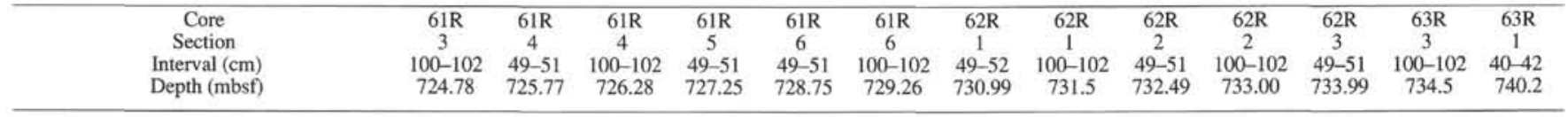

Sphaeroidinellopsis paenedehiscens

Sphaeroidinellopsis seminulina seminulina

Sphaeroidinellopsis kochi

Globigerinoides sacculifer

Neogloboquarina acostaensis

Globigerina (Z.) nepenthes

Pulleniatina primalis

Globorotalia (M.) multicamerata

Globorotalia (M.) menardii

Dentoglobigerina altispira altispira

Globorotalia (G.) tumida tumida

Globorotalia (G.) tumida flexuosa

Globorotalia (T.) crassula

Glbigerinoides conglobatus

Neogloboquadrina humerosa

Pulleniatina praecursor

Globigerinoides extremus

Sphaeroidinella dehiscens

Globorotalia (T.) crassaformis

Globigerinoides fistulosus

Globorotalia (T.) tosaensis

Globorotalia (T.) tosaensis tenuitheca

Globorotalia (T.) crassaformis ronda

Pulleniatina obliquiloculata

Globorotalia (T.) truncatulinoides

Diversity
Preservation

F

$\begin{array}{lllll}\text { F } & \text { F } & \text { F } & \text { F } & \text { F }\end{array}$

$\begin{array}{lll}\mathrm{F} & \mathrm{C} \\ \mathrm{C} & \mathrm{A} & \mathrm{C}\end{array}$

$\begin{array}{lll}\text { A } & \text { A } & \text { A } \\ \text { A } & \text { A } & \text { A } \\ \text { C } & & \text { C } \\ \text { C } & \text { C } & \text { C } \\ \text { C } & \text { F } & \text { R }\end{array}$

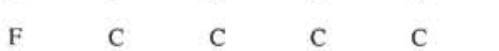

$\begin{array}{lllll}\mathrm{F} & \mathrm{C} & \mathrm{C} & \mathrm{C} & \mathrm{C} \\ \mathrm{A} & \mathrm{A} & \mathrm{A} & \mathrm{A} & \mathrm{A} \\ \mathrm{A} & \mathrm{A} & \mathrm{C} & \mathrm{C} & \mathrm{A}\end{array}$

C F

$\begin{array}{lll}\text { C } & \text { F } & \text { C } \\ \text { C } & & \text { C } \\ \text { A } & \text { A } & \text { A } \\ \text { C } & \text { C } & \text { C }\end{array}$

C
A
C

$\begin{array}{ll}\text { C } & \text { A } \\ \text { A } & \text { A } \\ \text { C } & \text { A } \\ \text { A } & \\ \text { C } & \text { C } \\ \text { R } & \text { R } \\ \text { C } & \text { C } \\ \text { A } & \text { A } \\ \text { C } & \text { A } \\ \text { C } & \text { C } \\ \text { A } & \text { A } \\ \text { C } & \text { C } \\ \text { A } & \text { C } \\ \text { H } & \text { H } \\ \text { G } & \text { G }\end{array}$

$\begin{array}{lll}\mathrm{A} & \mathrm{A} & \mathrm{A} \\ \mathrm{A} & \mathrm{A} & \mathrm{A} \\ \mathrm{A} & \mathrm{A} & \mathrm{C} \\ \mathrm{C} & \mathrm{C} & \mathrm{C} \\ \mathrm{F} & \mathrm{F} & \\ \mathrm{A} & \mathrm{C} & \mathrm{C} \\ \mathrm{A} & \mathrm{A} & \mathrm{A} \\ \mathrm{A} & \mathrm{C} & \mathrm{A} \\ \mathrm{F} & \mathrm{F} & \mathrm{A} \\ \mathrm{F} & \mathrm{F} & \mathrm{A} \\ \mathrm{C} & & \mathrm{C} \\ \mathrm{C} & & \\ \mathrm{H} & \mathrm{H} & \mathrm{H} \\ \mathrm{G} & \mathrm{G} & \mathrm{G}\end{array}$

${ }_{\mathrm{A}}^{\mathrm{C}}$

C $\quad \mathrm{C} \quad \mathrm{C} \quad \mathrm{F}$

Table 1 (continued).

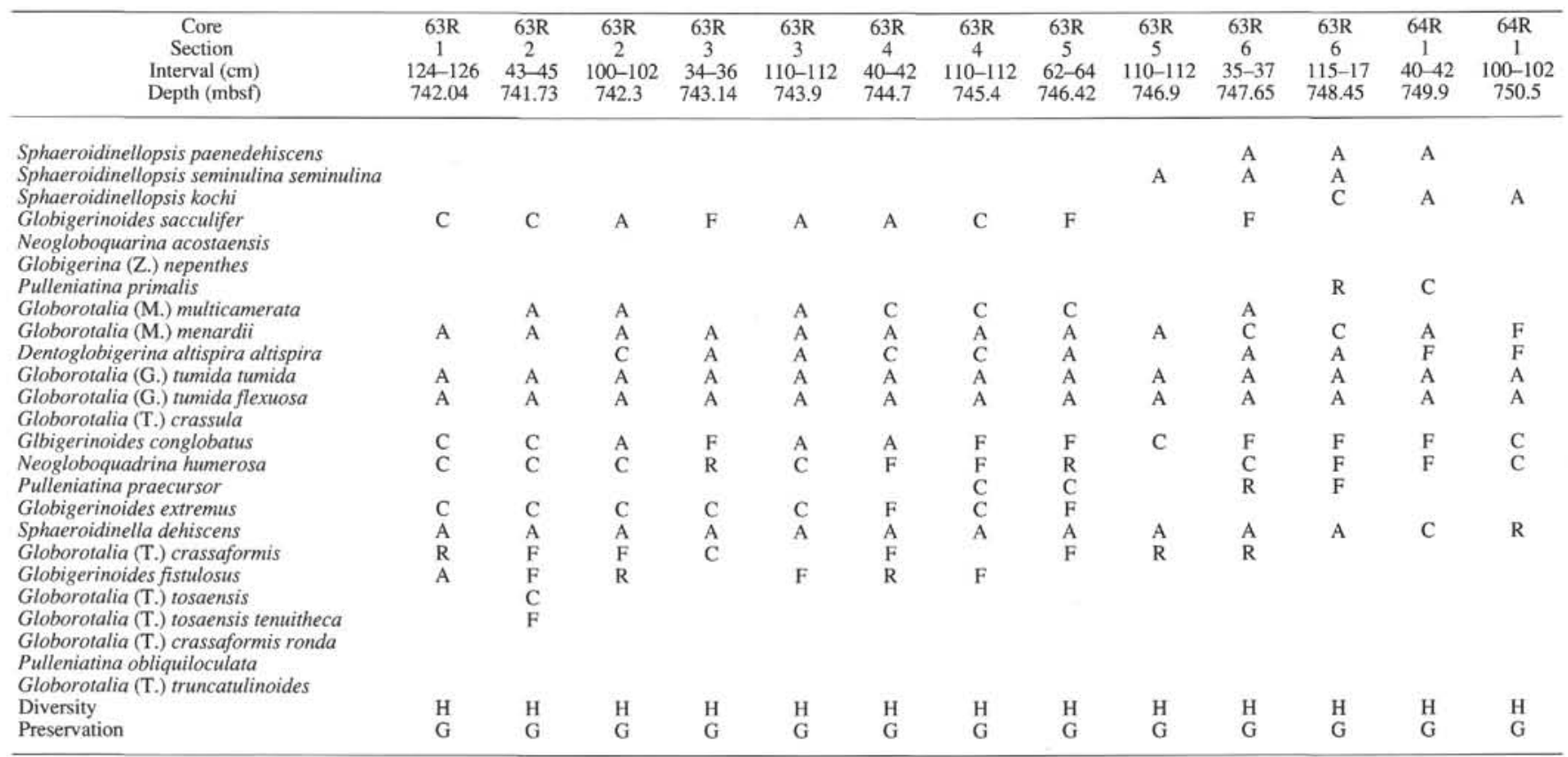

Zone N.22, and from DSDP Sites 209 and 210 (Coral Sea) near the base of Zone N.21. The FAD of $P$. obliquiloculata is diachronous within the southwest Pacific region.

In Hole 832B, the FAD of G. fistulosus occurs near the top of Zone N.20. Berggren et al. (1985) estimated an age of 2.9 Ma for this event within Zone N.21. Kennett and Srinivasan (1983) placed the FAD of G. fistulosus also in the middle part of Zone N.21; whereas Kennett (1973) recorded the event from DSDP Site 203 near the base of Zone N.22, from Site 209 in the lower part of Zone N.20, and from Site 210 in the middle part of Zone N.21. According to Kennett and Srinivasan (1983) G. fistulosus has a very short stratigraphic range (within Zone N.21). It occurs over a short interval between 733.00 and $743.90 \mathrm{mbsf}$ in Hole 832B. Its occurrence within the upper part of Zone N.20 at this site may be due to downhole contamination as the sediments are disturbed by numerous fractures and are heavily bioturbated (Collot, Greene, Stokking, et al., 1992, p. 467, 924). If contamination is the cause of the early incoming of $G$. fistulosus in the study area, then the FAD of $G$. fistulous may be reliable for the time correlation in the southwestern Pacific.

At Site 832, the FAD of $P$. praecursor lies within Zone N.18, below the FAD of $S$. dehiscens at the Zone N.19/N.18 boundary and above the FAD of $G$. (G.) tumida tumida at the Zone N.18/N.17B boundary. Kennett and Srinivasan (1983) placed the FAD of $P$. praecursor near the base of Zone N.19. Its position in Hole 832B may be because of 
Table 1 (continued).

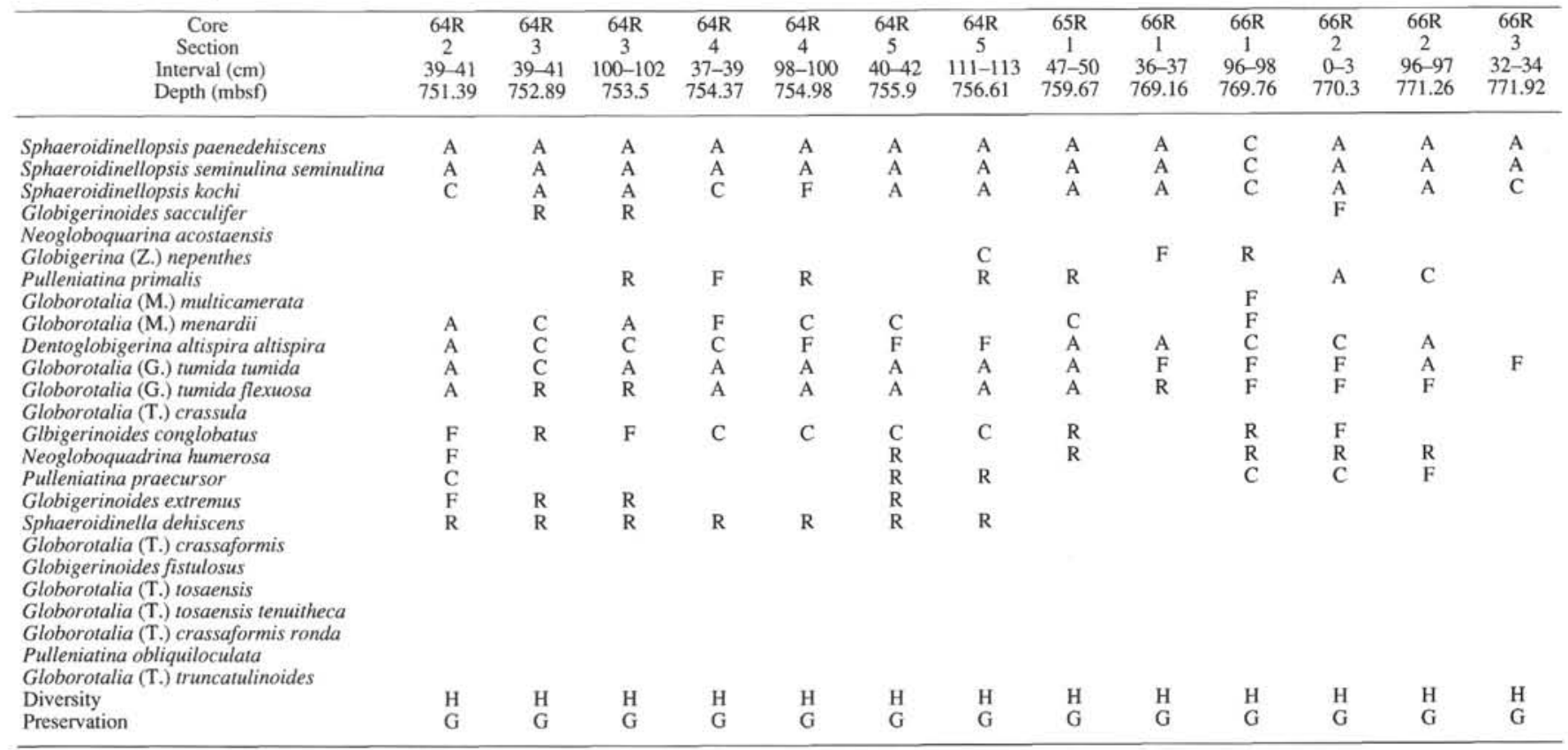

Table 1 (continued).

\begin{tabular}{|c|c|c|c|c|c|c|c|c|c|c|c|c|c|}
\hline $\begin{array}{c}\text { Core } \\
\text { Section } \\
\text { Interval }(\mathrm{cm}) \\
\text { Depth (mbsf) }\end{array}$ & $\begin{array}{c}66 \mathrm{R} \\
3 \\
92-94 \\
772.52\end{array}$ & $\begin{array}{c}66 \mathrm{R} \\
4 \\
1-3 . \\
773.11\end{array}$ & $\begin{array}{c}66 \mathrm{R} \\
4 \\
56-59 \\
773.66\end{array}$ & $\begin{array}{c}67 \mathrm{R} \\
1 \\
32-35 \\
778.92\end{array}$ & $\begin{array}{c}\text { 67R } \\
1 \\
117-118 \\
779.77\end{array}$ & $\begin{array}{c}67 R \\
2 \\
29-31 \\
780.39\end{array}$ & $\begin{array}{c}67 \mathrm{R} \\
2 \\
97-99 \\
781.07\end{array}$ & $\begin{array}{c}69 \mathrm{R} \\
1 \\
52-54 \\
798.32\end{array}$ & $\begin{array}{c}69 \mathrm{R} \\
1 \\
84-86 \\
798.64\end{array}$ & $\begin{array}{c}69 \mathrm{R} \\
2 \\
52-54 \\
799.82\end{array}$ & $\begin{array}{c}69 \mathrm{R} \\
2 \\
122-123 \\
800.52\end{array}$ & $\begin{array}{l}69 \mathrm{R} \\
3 \\
50-52 \\
801.3\end{array}$ & $\begin{array}{c}69 \mathrm{R} \\
4 \\
145-147 \\
803.75\end{array}$ \\
\hline Sphaeroidinellopsis paenedehiscens & $\mathrm{F}$ & $\mathrm{F}$ & C & A & C & A & A & A & A & A & A & A & A \\
\hline Sphaeroidinellopsis seminulina seminulina & $\mathrm{F}$ & F & $\mathrm{C}$ & $\mathrm{A}$ & $\mathrm{C}$ & $\mathrm{A}$ & A & A & A & A & A & A & A \\
\hline Sphaeroidinellopsis kochi & $\mathrm{F}$ & $\mathrm{F}$ & $\mathrm{C}$ & $\mathrm{A}$ & $\mathrm{F}$ & $\mathrm{F}$ & A & A & A & A & $\mathrm{A}$ & A & A \\
\hline $\begin{array}{l}\text { Globigerinoides sacculifer } \\
\text { Neogloboquarina acostaensis }\end{array}$ & & & & & & & & & & $\mathrm{F}$ & C & $\mathrm{F}$ & \\
\hline Globigerina (Z.) nepenthes & & & & C & $\mathrm{F}$ & F & & $\mathrm{F}$ & $\mathrm{F}$ & $\mathrm{F}$ & $\mathrm{F}$ & F & $\mathrm{C}$ \\
\hline Pulleniatina primalis & & & $\mathrm{C}$ & & $\mathrm{F}$ & $\mathrm{F}$ & $\mathrm{F}$ & C & $\mathrm{C}$ & $\mathrm{F}$ & A & A & $\mathrm{C}$ \\
\hline Globorotalia (M.) multicamerata & & & & & & & & & & $\mathrm{F}$ & $\mathrm{F}$ & & \\
\hline Globorotalia (M.) menardii & $\mathrm{F}$ & $\mathbf{R}$ & & C & $\mathrm{F}$ & $\mathrm{F}$ & $\mathrm{F}$ & $\mathrm{F}$ & $R$ & C & $\mathrm{C}$ & $\mathrm{C}$ & $\mathrm{C}$ \\
\hline Dentoglobigerina altispira altispira & & & & & A & A & C & $\mathrm{F}$ & C & A & & & \\
\hline Globorotalia (G.) tumida tumida & $\mathrm{F}$ & & $\mathrm{R}$ & A & $\mathrm{C}$ & A & A & C & $\mathrm{C}$ & A & A & $\mathrm{F}$ & \\
\hline $\begin{array}{l}\text { Globorotalia (G.) tumida flexuosa } \\
\text { Globorotalia (T.) crassula }\end{array}$ & & & $\mathrm{R}$ & A & & A & $\mathrm{F}$ & $\mathrm{F}$ & $\mathrm{C}$ & $\begin{array}{l}\mathrm{A} \\
\mathrm{R}\end{array}$ & A & & \\
\hline Glbigerinoides conglobatus & & & & C & & & $\mathrm{F}$ & $\mathrm{R}$ & $\mathrm{R}$ & $\mathrm{R}$ & $\mathrm{C}$ & & \\
\hline Neogloboquadrina humerosa & & & & C & & $\mathrm{C}$ & C & $\mathrm{C}$ & $\mathrm{F}$ & $\mathrm{F}$ & $\mathrm{F}$ & $\mathbf{R}$ & \\
\hline Pulleniatina praecursor & & & & & & & & $\mathbf{R}$ & $\mathrm{C}$ & $\mathrm{F}$ & F & A & \\
\hline Globigerinoides extremus & & & & & & & $\mathrm{F}$ & $\mathrm{F}$ & & & & & \\
\hline Sphaeroidinella dehiscens & & & & & & & & & & & & & \\
\hline Globorotalia (T.) crassaformis & & & & & & & & & & & & & \\
\hline Globigerinoides fistulosus & & & & & & & & & & & & & \\
\hline Globorotalia (T.) tosaensis & & & & & & & & & & & & & \\
\hline Globorotalia (T.) tosaensis tenuitheca & & & & & & & & & & & & & \\
\hline Globorotalia (T.) crassaformis ronda & & & & & & & & & & & & & \\
\hline Pulleniatina obliquiloculata & & & & & & & & & & & & & \\
\hline $\begin{array}{l}\text { Globorotalia }(\mathrm{T} .) \text { truncatulinoides } \\
\text { Diversity }\end{array}$ & & & & & & & & & & & $\mathrm{H}$ & & $\mathrm{H}$ \\
\hline $\begin{array}{l}\text { Diversity } \\
\text { Preservation }\end{array}$ & $\mathrm{L}$ & $\mathrm{L}$ & $\mathrm{H}$ & $\mathrm{H}$ & $\mathrm{H}$ & $\mathrm{H}$ & $\mathrm{H}$ & H & $\begin{array}{l}\mathrm{H} \\
\mathrm{G}\end{array}$ & $\begin{array}{l}\mathrm{H} \\
\mathrm{G}\end{array}$ & $\begin{array}{l}\mathrm{H} \\
\mathrm{G}\end{array}$ & $\begin{array}{l}\mathrm{H} \\
\mathrm{G}\end{array}$ & $\begin{array}{l}\text { H } \\
\text { G }\end{array}$ \\
\hline Preservation & G & G & G & G & G & G & G & G & $\mathrm{G}$ & U & & & \\
\hline
\end{tabular}

sediment disturbance caused by bioturbation and fracturing (as described for this part of the hole by Collot, Greene, Stokking, et al. 1992 , p. 468,930 ).

The FAD of $G$. (G.) tumida tumida at the Zone N.18/N.17B boundary is coincident with the FAD of $G$. (G.) tumida flexuosa within Hole 832B. Kennett and Srinivasan (1983) suggested that the FAD of G. (G.) tumida flexuosa occurred at the basal boundary of Zone N.19 above the FAD of $G$. (G.) tumida tumida. The FAD of $G$. (G.) tumida flexuosa in Hole 832B may be questionable because of taxonomic reasons. The subspecies is distinguished from $G$. $(G$.) tumida tumida by curvature of the final chamber. In studied assemblages typical $G$. (G.) tumida tumida (Plate 2, Fig. 4) morphologically intergrades with specimens showing variable flexure of the final chamber (e.g., Plate 2, Fig. 5). In light of the Shaw plot by Srinivasan and Sinha (1991), the FAD of $G$. $(G$.) tumida tumida appears synchronous in low-latitude areas of the southwest Pacific region, and therefore this datum level may be useful for time correlation in the studied region.

\section{Last Appearance Datum Levels}

The following species have anomalous last appearances in Hole 832B when compared to stratigraphic ranges recorded by Kennett and Srinivasan (1983): D. altispira altispira (in Zone N.20 rather than in Zone N.21), P. praecursor (in Zone N.20 rather than in Zone N.21), 
Table 1 (continued).

\begin{tabular}{|c|c|c|c|c|c|c|c|c|c|c|c|c|c|}
\hline $\begin{array}{c}\text { Core } \\
\text { Section } \\
\text { Interval }(\mathrm{cm}) \\
\text { Depth (mbsf) }\end{array}$ & $\begin{array}{c}69 \mathrm{R} \\
5 \\
56-58 \\
804.36\end{array}$ & $\begin{array}{c}69 \mathrm{R} \\
5 \\
110-112 \\
805\end{array}$ & $\begin{array}{c}70 \mathrm{R} \\
1 \\
13-15 \\
807.83\end{array}$ & $\begin{array}{c}70 \mathrm{R} \\
1 \\
149-150 \\
809.19\end{array}$ & $\begin{array}{c}70 \mathrm{R} \\
2 \\
26-28 \\
809.46\end{array}$ & $\begin{array}{c}70 \mathrm{R} \\
2 \\
103-106 \\
810.23\end{array}$ & $\begin{array}{c}70 \mathrm{R} \\
3 \\
45-49 \\
811.15\end{array}$ & $\begin{array}{c}70 \mathrm{R} \\
3 \\
148-150 \\
812.18\end{array}$ & $\begin{array}{c}70 \mathrm{R} \\
4 \\
24-27 \\
812.44\end{array}$ & $\begin{array}{c}70 \mathrm{R} \\
4 \\
92-95 \\
813.12\end{array}$ & $\begin{array}{c}70 \mathrm{R} \\
5 \\
44.47 \\
814.14\end{array}$ & $\begin{array}{c}70 \mathrm{R} \\
5 \\
145-147 \\
815.15\end{array}$ & $\begin{array}{c}70 R \\
6 \\
48-51 \\
815.68\end{array}$ \\
\hline Sphaeroidinellopsis paenedehiscens & A & A & A & A & A & A & A & A & $\mathrm{A}$ & A & A & A & A \\
\hline Sphaeroidinellopsis seminulina seminulina & A & $\mathrm{A}$ & $\mathrm{A}$ & $\mathrm{A}$ & $\mathrm{A}$ & $\mathrm{A}$ & $\mathrm{A}$ & A & $\mathrm{A}$ & A & $\mathrm{A}$ & A & A \\
\hline Sphaeroidinellopsis kochi & A & $\mathrm{A}$ & $\mathrm{A}$ & A & A & A & A & A & A & C & C & C & A \\
\hline \multicolumn{14}{|l|}{ Neogloboquarina acostaensis } \\
\hline Globigerina (Z.) nepenthes & $\mathrm{C}$ & $\mathrm{C}$ & $\mathrm{C}$ & $\mathrm{C}$ & $\mathrm{F}$ & $\mathrm{F}$ & $\mathrm{F}$ & $\mathrm{C}$ & $\mathrm{F}$ & $\mathrm{C}$ & A & $\mathrm{C}$ & $\mathrm{C}$ \\
\hline Pulleniatina primalis & A & $\mathrm{C}$ & $\mathrm{C}$ & $\mathrm{C}$ & $\mathrm{C}$ & $\mathrm{R}$ & & & $\mathrm{F}$ & $\mathrm{C}$ & $\mathrm{C}$ & A & $\mathrm{F}$ \\
\hline Globorotalia (M.) multicamerata & & C & A & A & $\mathrm{C}$ & A & $\mathrm{F}$ & A & A & A & A & $\mathrm{A}$ & \\
\hline Globorotalia (M.) menardii & $\mathrm{F}$ & $\mathrm{F}$ & A & C & & A & $\mathrm{C}$ & A & A & $\mathbf{R}$ & A & & \\
\hline Dentoglobigerina altispira altispira & & A & $\mathrm{C}$ & & & C & $\mathrm{C}$ & $\mathrm{F}$ & $\mathrm{F}$ & $\mathrm{A}$ & A & A & $\mathrm{C}$ \\
\hline Globorotalia (G.) tumida tumida & $\mathrm{C}$ & & A & A & A & A & A & & & & & & \\
\hline Globorotalia (G.) tumida flexuosa & C & & A & A & $\mathrm{A}$ & A & $\mathrm{C}$ & & & & & & \\
\hline Globorotalia (T.) crassula & $\mathrm{F}$ & A & $\mathbf{R}$ & C & & & & & & & & & \\
\hline Glbigerinoides conglobatus & $\mathrm{C}$ & $\mathrm{F}$ & $\mathrm{C}$ & $\mathrm{F}$ & & & & & & & & & \\
\hline Neogloboquadrina humerosa & $\mathrm{F}$ & $\mathrm{F}$ & & & & & & & & & & & \\
\hline \\
\hline \multirow{2}{*}{\multicolumn{14}{|c|}{ Globigerinoides extremus }} \\
\hline \multirow{2}{*}{\multicolumn{13}{|c|}{$\begin{array}{l}\text { Sphaeroidinella dehiscens } \\
\text { Globorotalia (T.) crassaformis }\end{array}$}} & \\
\hline & & & & & & & & & & & & & \\
\hline \multicolumn{14}{|l|}{ Globigerinoides fistulosus } \\
\hline \multicolumn{14}{|l|}{ Globorotalia (T.) tosaensis } \\
\hline \multicolumn{14}{|l|}{ Globorotalia (T.) tosaensis tenuitheca } \\
\hline \multirow{2}{*}{\multicolumn{14}{|c|}{$\begin{array}{l}\text { Globorotalia (T.) crassaformis ronda } \\
\text { Pulleniatina obliquiloculata }\end{array}$}} \\
\hline & & & & & & & & & & & & & \\
\hline \multicolumn{14}{|l|}{ Globorotalia (T.) truncatulinoides } \\
\hline Diversity & $\mathrm{H}$ & $\mathrm{H}$ & $\mathrm{H}$ & $\mathrm{H}$ & $\mathrm{H}$ & $\mathrm{H}$ & $\mathrm{H}$ & $\mathrm{H}$ & $\mathrm{H}$ & $\mathrm{H}$ & $\mathrm{H}$ & $\mathrm{H}$ & $\mathrm{H}$ \\
\hline Preservation & G & G & G & G & G & G & G & G & G & G & G & G & G \\
\hline
\end{tabular}

Table 1 (continued).

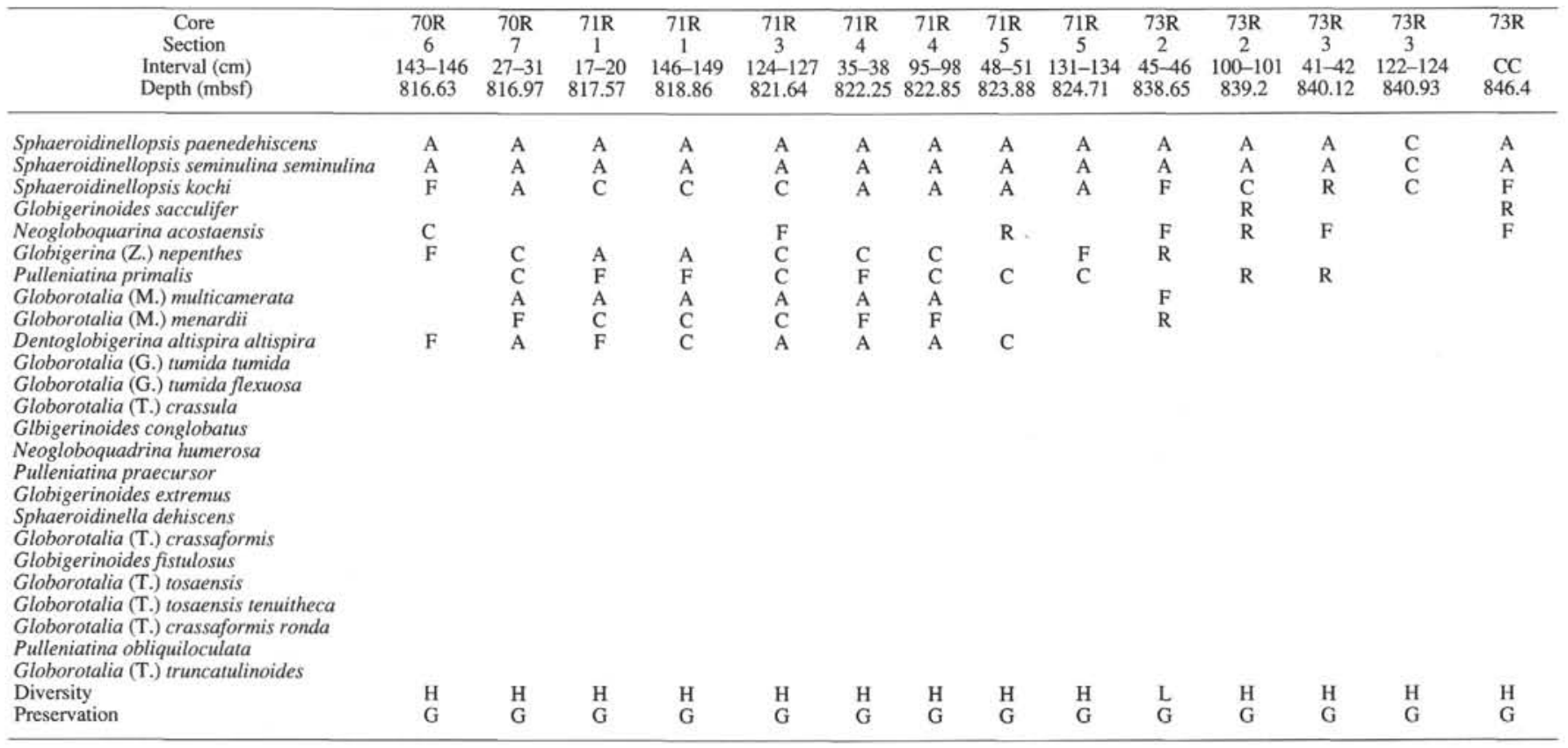

S. paenedehiscens (in Zone N.19 rather than in Zone N.20), S. seminulina seminulina (in Zone N.19 rather than in Zone N.21), G. (Z.) nepenthes (at the Zone N.19/N.18 boundary rather than in Zone N.20), P. primalis (in Zone N.19 rather than in Zone N.20), and N. acostaensis (in Zone N.17B rather than in Zone N.20), G. (G.) tumida flexuosa (in Zone N.22 rather than in Zone N.21), N. humerosa (in Zone N.22 rather than in Zone N.21).

At Site 832 the LAD of $D$. altispira altispira lies near the top of Zone N.20, just below the FAD of $G$. (T.) tosaensis at the Zone N.22/21 boundary, and approximates the LAD level recognized by Kennett and Srinivasan (1983). Chaproniere (1991) recorded this species occurring with $G$. (T.) truncatulinoides and suggested that it ranges to within the basal part of Zone N.22. Berggren et al. (1985) estimated an age of 2.9 Ma near the base of Zone N.21 for the LAD of
D. altispira altispira; Kennett and Srinivasan (1983) also placed the LAD of $D$. altispira altispira just above the base of Zone N.21; and Heath and McGowran (1984) recognized the datum within Zone N.21. Kennett (1973) recorded the LAD of D. altispira altispira from the DSDP Site 210 near the basal boundary of Zone N.22, from Site 209 in the upper part of Zone N.21, and from Site 203 just below the base of Zone N.21. Srinivasan and Sinha (1991) regarded the LAD of $D$. altispira altispira as a first-order datum and therefore suggested that it was nearly synchronous within tropical to warm subtropical latitudes. In the light of all the findings discussed above, the LAD of D. altispira altispira may be reliable for time correlation of a level near the N.21/ N.20 boundary, and the higher records may be due to reworking.

In Hole 832B, the LAD of $P$. praecursor lies within Zone N.20, below the FAD of G. (T.) tosaensis and above the FAD of $G$. (T.) cras- 


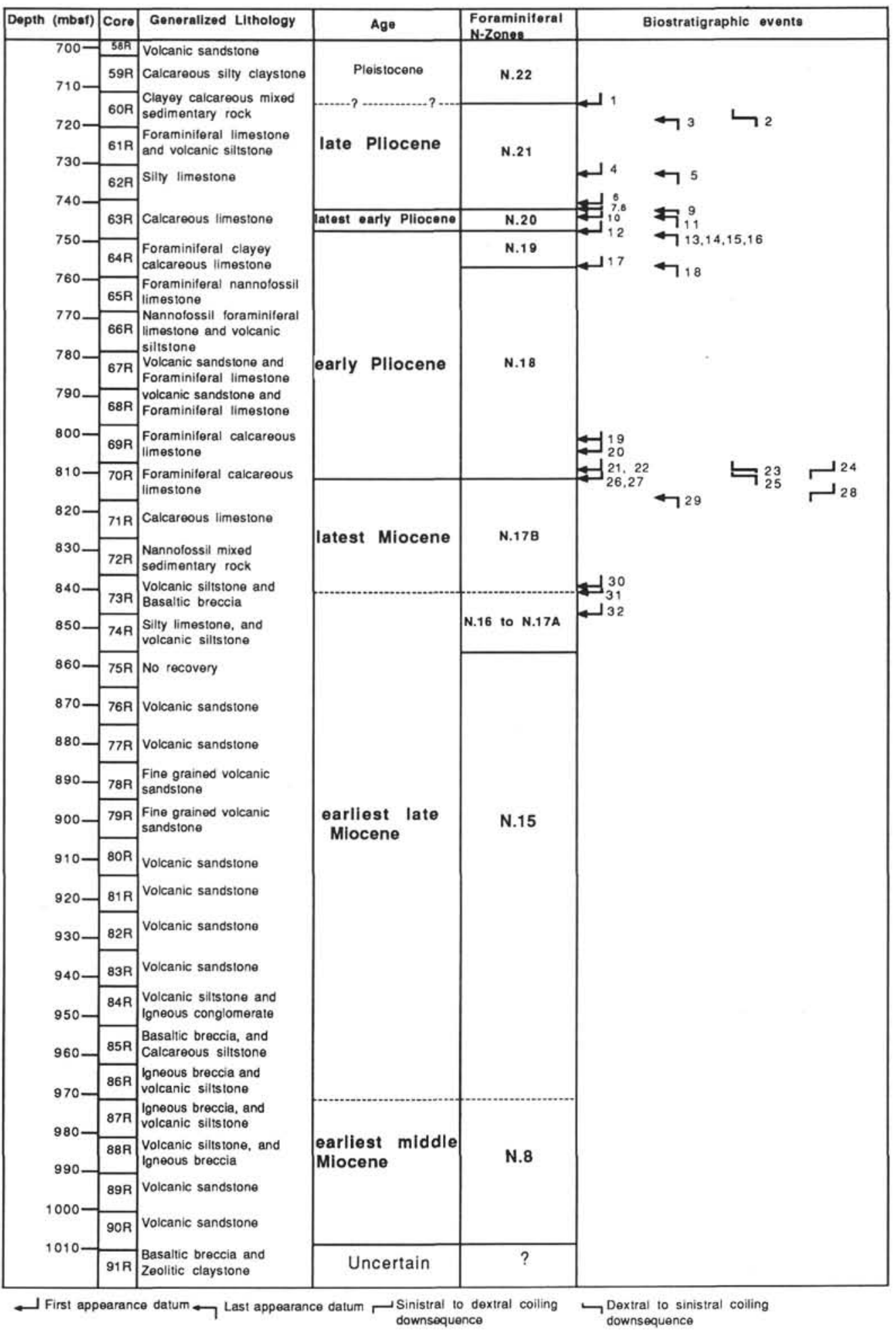

Figure 2. Stratigraphic distribution of important biostratigraphic events. The first appearance datum levels represented here are as follows: $1=$ Globorotalia (Truncorotalia) truncatulinoides (d'Orbigny), 4 = Pulleniatina obliquiloculata (Parker and Jones), $6=$ Globorotalia (Truncorotalia) crassaformis ronda (Blow; after Bolli and Saunders, 1985), 7 = Globorotalia (Truncorotalia) tosaensis (Takayanagi and Saito), $8=$ Globorotalia (Truncorotalia) tosaensis tenuitheca (Blow; after Bolli and Saunders, 1985), $10=$ Globigerinoides fistulosus (Schubert), $12=$ Globorotalia (Truncorotalia) crassaformis (Galloway and Wissler) s.s., $17=$ Sphaeroidinella dehiscens (Parker and Jones), $19=$ Pulleniatina praecursor Banner and Blow, $20=$ Neogloboquadrina humerosa $($ Takayanagi and Saito), $21=$ Globorotalia (Truncorotalia) crassula Cushman and Stewart, $26=$ Globorotalia (Globorotalia) tumida tumida $($ Brady), $27=$ Globorotalia $($ Globorotalia) tumida flexuosa $($ Koch), 30 = Globorotalia (Menardella) multicamerata Cushman and Jarvis, $31=$ Pulleniatina primalis Banner and Blow. Last appearance datum levels: $3=$ Globorotalia (Menardella) multicamerata, $5=$ Globigerinoides fistulosus, $9=$ Dentoglobigerina altispira altispira, $11=$ Pulleniatina praecursor, $13=$ Sphaeroidinellopsis paenedehiscens, $14=$ Sphaeroidinellopsis seminulina seminulina, $15=$ Sphaeroidinellopsis kochi, $16=$ Pulleniatina primalis, $18=$ Globigerina (Zeaglobigerina) nepenthes, $20=$ Neogloboquadrina acostaensis. Coiling changes: $2=$ Globorotalia (Menardella) menardii, $23=$ Globorotalia (Globorotalia) tumida flexuosa, 24 = Globorotalia (Menardella) multicamerata, 25, $28=$ Pulleniatina primalis. 
Table 2. Stratigraphic distribution of biostratigaphically important planktonic foraminiferal species from the lowest middle Miocene to upper Miocene; their relative abundance, diversity, and preservation are visually estimated, with relative abundance denoted as follows: $\mathrm{A}=$ abundant $=$ greater than 10 specimens, $\mathrm{C}=$ common $=6-10$ specimens, $\mathrm{F}=$ few $=3-5$ specimens, and $R=$ rare $=1-2$ specimens. Preservation is indicated thusly: $G=$ good, for specimens visually identifiable, $M=$ moderate, for specimens with some problems in distinguishing, and $\mathbf{P}=$ poor, which are very difficult to discriminate between individual taxa. Diversity in a total foraminiferal assemblage is estimated as high $(\mathrm{H})$ for greater than $\mathbf{5}$ species, and low $(\mathrm{L})$ for 1-5 species.

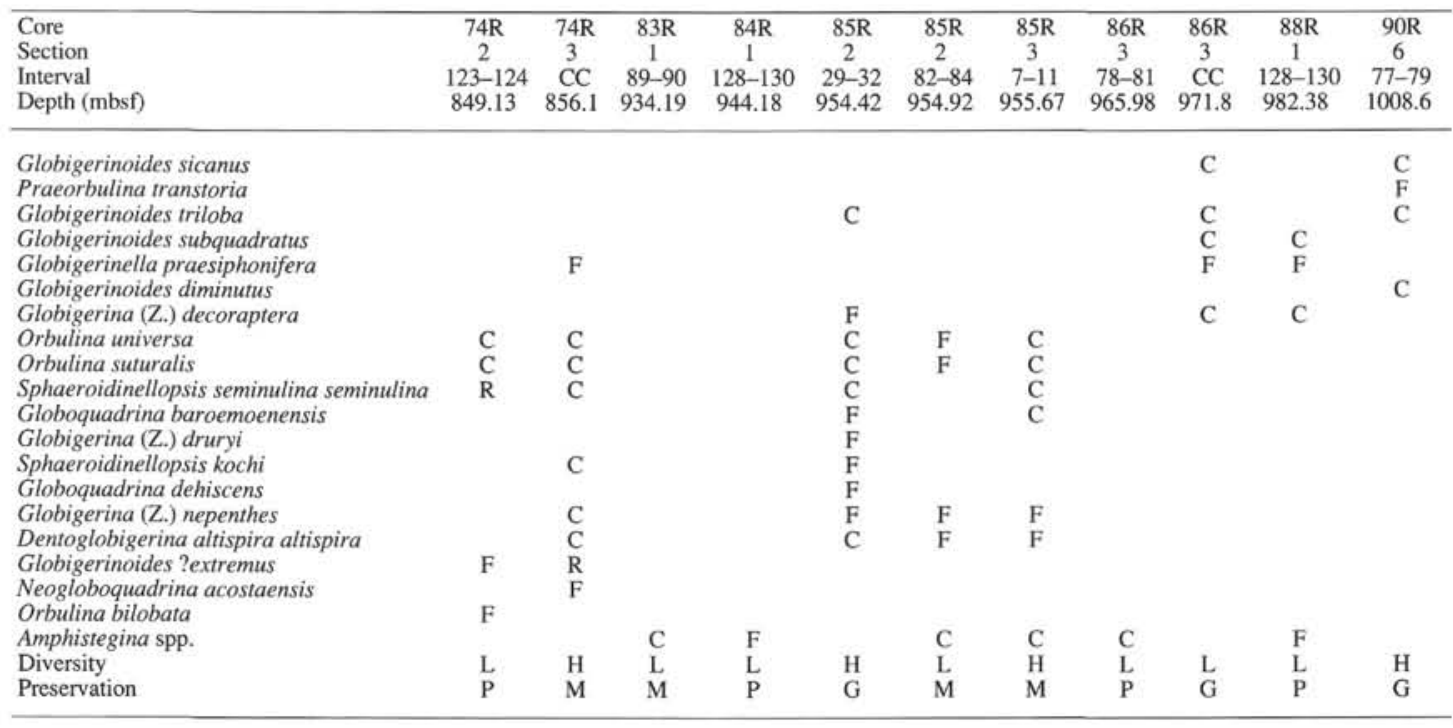

saformis s.s. Kennett and Srinivasan (1983) placed the LAD of $P$. praecursor near the top of Zone N.21, and Chaproniere (1991) placed this datum within Zone N.22. The LAD of $P$. praecursor therefore appears to be diachronous in the studied region. The ancestral $P$. praecursor gradually evolves into the more advanced $P$. obliquiloculata by becoming more involute, and therefore its LAD position is difficult to recognize at a consistent level.

The LADs of $S$. paenedehiscens and $S$. seminulina seminulina are located near the top of Zone N.19 at Site 832, below the FAD of $G$. (T.) crassaformis s.s. at the Zone N.20/N.19 boundary, below the LAD of $D$. altispira altispira and above the FAD of $S$. dehiscens at the Zone N.19/N.18 boundary. Berggren et al. (1985) estimated an age for the LAD of Sphaeroidinellopsis spp. at 3.0 Ma near the base of Zone N.21. In the range chart of Kennett and Srinivasan (1983), the LAD of $S$. paenedehiscens is positioned in the upper part of Zone N.19-20 (stated in their text as in Zone N.20), and the LAD of $S$. seminulina seminulina in the middle part of Zone N.21. Srinivasan and Sinha (1991) regarded the LAD of Sphaeroidinellopsis as nearly synchronous in the southwest Pacific region. However, the low position of the LADs of $S$. seminulina seminulina and $S$. paenedehiscens in Hole 832B when compared to other records in the western Pacific region suggest that these datum levels cannot be used for time correlation at the studied site.

The LAD of $P$. primalis is located near the top of Zone N.19 in Hole $832 \mathrm{~B}$, below the FAD of $G$. (T.) crassaformis s.s. at the Zone N.20/N.19 boundary, and above the FAD of S. dehiscens at the Zone N.19/N.18 boundary. Berggren et al. (1985) estimated an age for the LAD of $P$. primalis at 3.5 Ma within Zone N.20. Kennett and Srinivasan (1983) placed the LAD of P. primalis within Zone N.19-N.20 (stated in their text as Zone N.20). Pulleniatina primalis is distinguished as the ancestor of $P$. praecursor by its less developed cortex and less pronounced streptospiral coiling. Because it is difficult to distinguish these species in a consistent way at different sites, slight differences in the positioning of the LAD of $P$. primalis may occur in different studies.

In Hole 832B, the LAD of $G$. (Z.) nepenthes is located at the Zone N.19/N.18 boundary, well above the FAD of $G$. (G.) tumida tumida at the Zone N.18/N.17B boundary and coincident with the FAD of $S$. dehiscens. Berggren et al. (1985) estimated the LAD of G. (Z.) nepenthes at 3.9 Ma within Zone N.20. Kennett and Srinivasan (1983) placed the LAD of $G$. (Z.) nepenthes near the top of Zone N.19-20 (stated in their text as in Zone N.19). From DSDP Site 210, the LAD of $G$. (Z.) nepenthes was recorded in the upper part of Zone N.22 by Kennett (1973), whereas Heath and McGowran (1984) placed it in the upper part of Zone N.19 at Site 289. Srinivasan and Sinha (1991) showed the diachronous nature of the LAD of $G$. nepenthes in the low-latitude areas of the southwest Pacific, and therefore this datum is probably an unreliable time marker in the studied region.

The LAD of $N$. acostaensis lies near the top of Zone N.17B below the FAD of $G$. (G.) tumida tumida at the Zone N.18/N.17B boundary, and above the FAD of $P$. primalis at the Zone N.17B/N.17A boundary at Site 832. Kennett and Srinivasan (1983) placed the LAD of $N$. acostaensis near the top of Zone N.19-20 (stated in their text as in Zone N.20). Neogloboquadrina acostaensis is distinguished as the ancestor of $N$. humerosa by having less chambers in the final whorl, closed umbilicus, and a distinct apertural plate. Because it is difficult to distinguish these species in a consistent way at different sites, slight differences in the positioning of the LAD of $N$. acostaensis may occur in different studies.

The extended stratigraphic ranges of $G$. (G.) tumida flexuosa and $N$. humerosa in Hole 832B may be because of reworking in this tectonically active basin, or due to taxonomic problems similar to those discussed above for $P$. praecursor. Therefore the LADs of these species may be unreliable for time correlation in Hole 832B.

\section{Coiling Changes}

In Hole $832 \mathrm{~B}$, a coiling change downsequence from sinistral to dextral in the assemblages of $G$. $(M$.) menardii is located near the top of Zone N.21, below the FAD of $G$. (T.) truncatulinoides at the Zone N.22/N.21 boundary and above the FAD of $G$. (T.) tosaensis at the Zone N.21/N.20 boundary. Downsequence changes from sinistral to dextral coiling in G. tumida flexuosa and P. primalis, and a change from dextral to sinistral in the assemblages of $G$. (M.) multicamerata, lie within Zone N.18, just above the FAD of $G$. $(G$.) tumida tumida at the Zone N.18/N.17B boundary. Pulleniatina primalis again changes 
its coiling direction from dextral to sinistral downsequence within Zone N.17B, below the FAD of $G$. (G.) tumida tumida.

Although Heath and McGowran (1984) recognized in assemblages of Globorotalia cultrata s.l. and Globorotalia tumida s.l. changes downsequence from sinistral to dextral within zone N.17, and from dextral to sinistral near the base of zone N.17; much more data on the changes in the coiling directions of trochospiral species are required before these levels can be compared and evaluated for use for time correlation in the southwest Pacific region.

\section{AGE OF UNITS AND DISCUSSION}

At Site 832, the zonal index species (e.g., G. (T.) truncatulinoides, $G$. (T.) tosaensis, G. (T.) crassaformis s.s., S. dehiscens, $G$. (G.) tumida tumida, $P$. primalis, $N$. acostaensis, $G$. nepenthes and $G$. sicanus) are found in their expected stratigraphic order. Therefore the borehole succession can be subdivided into planktonic foraminiferal zones widely recognized throughout the southwest Pacific (as discussed above). An evaluation of 32 datum levels suggests that the following may be the most reliable tie points to the chronometric scale of Berggren et al. (1985): FADs of G. (T.) truncatulinoides (1.9 Ma), G. (T.) tosaensis (3.1 Ma), G. (T.) crassaformis (4.3 Ma), S. dehiscens (5.1 Ma), G. (G.) tumida tumida (5.2 Ma), G. conglobatus (5.3 Ma), and $P$. primalis $(5.8 \mathrm{Ma})$; and LAD of $G$. (M.) multicamerata $(2.9$ $\mathrm{Ma})$, and $D$. altispira altispira (2.9 Ma).

Based on the zonal correlation shown in Figure 2, the foraminiferal limestone and claystone of lithostratigraphic Unit V from 702 to $714.10 \mathrm{mbsf}$ is probably Pleistocene or late Pliocene in age. The age of the lithostratigraphic Unit V from 714.10 to $741.73 \mathrm{mbsf}$ is late Pliocene, 741.73 to $747.65 \mathrm{mbsf}$ is latest early Pliocene, 747.65 to 811.15 mbsf is early Pliocene, 811.15 to 840.93 mbsf is latest Miocene, and from 840.93 to $865.7 \mathrm{mbsf}$ is earliest late Miocene in age. Based on the chronometry of datum levels listed above, lithostratigraphic Unit $\mathrm{V}$ accumulated at about $30 \mathrm{~m} / \mathrm{m}$.y. However this may be an underestimation because a number of datum levels ranging from 2.9 Ma to 5.1 Ma occur within a structurally deformed zone between 740 and 760 mbsf.

Lithostratigraphic Unit VI from 865.7 to $952.6 \mathrm{mbsf}$ and lithostratigraphic Unit VII from 952.6 to 971.8 mbsf are earliest late Miocene in age. The oldest sediments in Hole $832 \mathrm{~B}$ belong to lithostratigraphic Unit VII, mainly volcanic sandstone, from 971.8 to 1106.7 mbsf. Samples collected below $1008.6 \mathrm{mbsf}$ did not yield foraminifera and therefore the age of the lowest portion of the cored section is indeterminate, whereas similar sediments between 1008.6 to $971.80 \mathrm{mbsf}$ contain a sparse assemblage of planktonic foraminifera indicating an earliest middle Miocene age. This determination is supported by the occurrence of larger benthonic foraminifers Lepidocyclina sp., Miogyspina spp, and Amphistegina spp. within this interval. The larger foraminifers are indicative of lower Tf Letter Stage (Adams, 1984) and are considered contemporaneous with basin deposition but transported from an adjacent neritic environment.

The abrupt increase during the latest Miocene in abundance upsequence of planktonic foraminifers (at $846.40 \mathrm{mbsf}$ ) corresponds with the increase in calcareous sediment, and may indicate either deepening of the basin, increase in productivity of calcareous plankton, decrease in volcanoclastic sedimentation, or a combination of these. The introduction of warmer surface water conditions may be reflected by the incoming of abundant keeled Globorotalia close to the Miocene/Pliocene boundary (at $822.85 \mathrm{mbsf}$ ).

\section{CONCLUSIONS}

The interval between 702 and 1008.6 mbsf in Hole $832 \mathrm{~B}$ is subdivided downsequence into Zones N.22, N.21, N.20, N.19, N.18, N.17B, N.16 to N.17A, N.15, and N.8. In the planktonic foraminiferal succession in Zones N.22 through N.17B, 32 biostratigraphic events are recognized, and these are evaluated to determine their significance for time correlation. The positions of some datum levels in Hole 832B seem to have been affected by sediment mixing due to bioturbation or to structural disturbance (particularly between 740 and $760 \mathrm{mbsf}$ ). Therefore some of the first and last appearances of species in the hole may be diachronous when compared to these events elsewhere in the western Pacific.

Zonation and age determination of the borehole reveals the following:

1. The oldest sediments are mainly volcanic sandstone of earliest middle Miocene (planktonic foraminiferal Zone N.8) age or older.

2. An unconformity appears to be present near $971.80 \mathrm{mbsf}$ and includes the Zone N.9 through N.14 interval (i.e., most of the middle Miocene), which has not been located in the drill section.

3. A change in depositional conditions occurred during the latest Miocene (at $846.40 \mathrm{mbsf}$, within Zone N.17), when an abundant assemblage of planktonic foraminifera first appears in the region.

4. During the latest Miocene through earliest Pliocene (planktonic foraminiferal Zones N.17B and N.22) a continuous stratigraphic sequence of mainly calcareous sediment accumulated at a rate of about $30 \mathrm{~m} / \mathrm{m} . \mathrm{y}$.

\section{ACKNOWLEDGMENTS}

I wish to thank Dr. D.W. Haig for the guidance and encouragement he gave me during preparation of this manuscript, and George Chaproniere and Greg Milner for reviewing the manuscript. The Center for Microscopy and Microanalysis of the University of Western Australia facilitated my use of a scanning electron microscope. I am grateful to SOPAC and the University of Western Australia for providing funds that enabled me to contribute to Leg 134. I am grateful to the Australian International Development Assistance Bureau for supporting my studies at the University of Western Australia.

\section{REFERENCES}

Adams, C.G., 1984. Neogene larger foraminifera, evolutionary and geological events in the context of datum planes. In Ikebe, N., and Tsuchi, R. (Eds.), Pacific Neogene Datum Planes: Contributions to Biostratigraphy and Chronology: Tokyo (Univ. of Tokyo Press), 47-67.

Berggren, W.A., Kent, D.V., and Van Couvering, J.A., 1985. The Neogene: Part 2. Neogene geochronology and chronostratigraphy. In Snelling, N.J. (Ed.), The Chronology of the Geological Record. Geol. Soc. London Mem., 10:211-260.

Blow, W.H., 1969. Late middle Eocene to Recent planktonic foraminiferal biostratigraphy. In Brönniman, P., and Renz, H.H. (Eds.), Proc. First Int. Conf. Planktonic Microfossils, Geneva, 1967: Leiden (E.J. Brill), 1:199422.

Bolli, H.M., and Saunders, J.B., 1985. Oligocene to Holocene low latitude planktonic foraminifera. In Bolli, H.M., Saunders, J.B., and Perch-Nielsen, K. (Eds.), Plankton Stratigraphy: Cambridge (Cambridge Univ. Press), $155-262$.

Brönnimann, P., and Resig, J., 1971. A Neogene globigerinacean biochronologic time-scale of the Southwestern Pacific. In Winterer, E.L., Riedel, W.R., et al., Init. Repts. DSDP, 7 (Pt. 2): Washington (U.S. Govt. Printing Office), 1235-1469.

Chaproniere, G.C.H., 1991. Pleistocene to Holocene planktic foraminiferal biostratigraphy of the Coral Sea, offshore Queensland, Australia. BMR J. Aust. Geol. Geophys., 12:195-221.

Haig, D.W., and Perembo, R.C.B., 1990. Foraminifera as stratigraphic guides for Papua New Guinea. In Carmen, G.J., and Carmen, Z. (Eds.), Petroleum Exploration in Papua New Guinea: Proceedings of the First PNG Petroleum Convention. Port Moresby, 381-395.

Heath, R.S., and McGowran, B., 1984. Neogene datum planes: foraminiferal successions in Australia with reference sections from the Ninetyeast Ridge and the Ontong-Java Plateau. In Ikebe, N., and Tsuchi, R. (Eds.), Pacific

\footnotetext{
- Abbreviations for names of organizations and publications in ODP reference lists follow the style given in Chemical Abstracts Service Source Index (published by American Chemical Society).
} 
Neogene Datum Planes: Contributions to Biostratigraphy and Chronology: Tokyo (Univ. of Tokyo Press), 187-191.

Hills, S.J., and Thierstein, H.R., 1989. Plio-Pleistocene calcareous plankton biochronology. Mar. Micropaleontol., 14:67-96.

Kennett, J.P., 1973. Middle and late Cenozoic planktonic foraminiferal biostratigraphy of the Southwest Pacific - DSDP Leg 21. In Burns, R.E., Andrews, J.E., et al., Init. Repts. DSDP, 21: Washington (U.S. Govt. Printing Office), 575-639.

Kennett, J.P., Keller, G., and Srinivasan, M.S., 1985. Miocene planktonic foraminiferal biogeography and paleoceanographic development of the Indo-Pacific region. In Kennett, J.P. (Ed.), The Miocene Ocean: Paleoceanography and Biogeography. Mem.-Geol. Soc. Am., 163:197-236.

Kennett, J.P., and Srinivasan, M.S., 1983. Neogene Planktonic Foraminifera: A Phylogenetic Atlas: Stroudsburg, PA (Hutchinson Ross).

1984. Neogene datum planes: foraminiferal successions in Australia with reference sections from the Ninetyeast Ridge and the Ontong-Java Plateau. In Ikebe, N., and Tsuchi, R. (Eds.), Pacific Neogene Datum Planes, Contributions to Biostratigraphy and Chronology: Tokyo (Univ. of Tokyo Press), 11-33.
Srinivasan, M.S., and Kennett, J.P., 1981a. A review of Neogene planktonic foraminiferal biostratigraphy: applications in the equatorial and South Pacific. In Warme, J.E., Douglas, R.G., and Winterer, E.L. (Eds.), The Deep Sea Drilling Project: A decade of progress. SEPM Special Publication, 32:395-432.

Srinivasan, M.S., and Kennett, J.P., 1981b. Neogene planktonic foraminiferal biostratigraphy and evolution: equatorial to subantarctic, South Pacific. Mar. Micropaleontol., 6:499-533.

Srinivasan, M.S., and Sinha, D.K., 1991. Improved correlation of the late Neogene planktonic foraminiferal datum in the equatorial to cool subtropical DSDP Sites, Southwest Pacific: application of the graphic correlation method. In Radhakrishna, B.P. (Ed.), The World of Martin F. Glaessner. Mem.-Geol. Soc. India, 20:55-93.

Date of initial receipt: 10 June 1992

Date of acceptance: 25 March 1993

Ms 134SR-010 


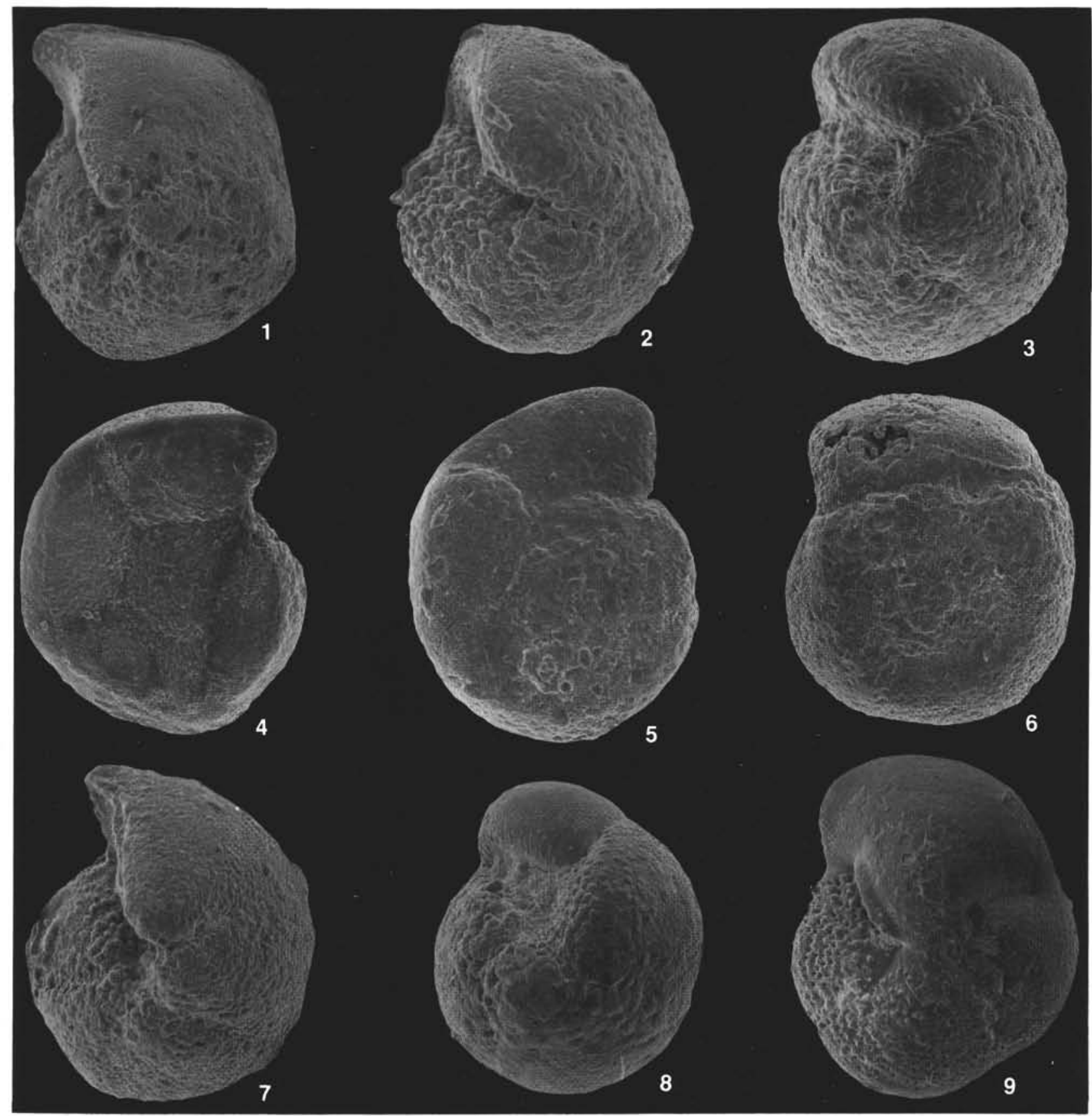

Plate 1. Biostratigraphically important species in sediments from the uppermost Miocene to upper Pliocene. 1, 4. Globorotalia (Truncorotalia) truncatulinoides (d'Orbigny), (1) umbilical view, $\times 80,134-832 \mathrm{~B}-59 \mathrm{R}-4,99-101 \mathrm{~cm},(4)$ spiral view, $\times 107,134-832 \mathrm{~B}-59 \mathrm{R}-4,99-101 \mathrm{~cm} . \quad 2,5$. Globorotalia (Truncorotalia) tosaensis (Takayanagi and Saito), (2) umbilical view, $\times 107,134-832 \mathrm{~B}-60 \mathrm{R}-4,50-51 \mathrm{~cm},(5) \mathrm{spiral}$ view, $\times 134$, 134-832B-60R-4-50-51 cm. 3, 6. Globorotalia (Truncorotalia) crassaformis s.s. (Galloway and Wissler), (3) umbilical view, $\times 107,134-832 \mathrm{~B}-61 \mathrm{R}-1$, 100-102 cm, (6) spiral view, $\times 93,134-832 \mathrm{~B}-61 \mathrm{R}-1,100-102 \mathrm{~cm} . \quad 7$. Globorotalia (Truncorotalia) tosaensis tenuitheca (Blow), umbilical view, $\times 93$, 134-832B-59R-4, 49-51 cm. 8. Globorotalia (Truncorotalia) crassaformis ronda (Blow), umbilical view, $\times 93,134-832 \mathrm{~B}-59 \mathrm{R}-1,99-101 \mathrm{~cm} .9$. Globorotalia (Truncorotalia) crassula Blow, umbilical view, $\times 134,134-832 \mathrm{~B}-59 \mathrm{R}-1,99-101 \mathrm{~cm}$. 


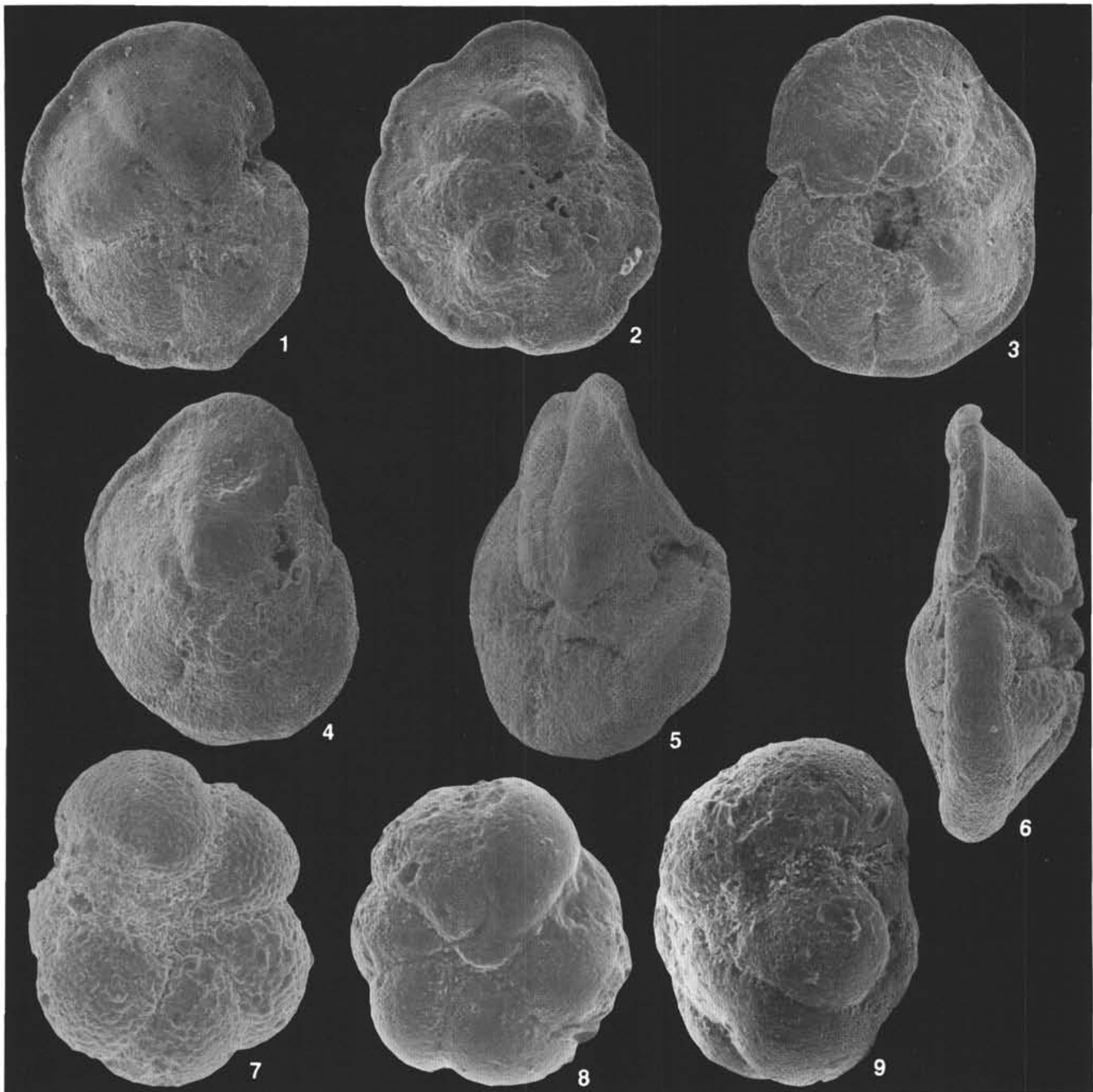

Plate 2. Biostratigraphically important species in sediments from the uppermost Miocene to upper Pliocene. 1. Globorotalia (Menardella) menardii (Parker, Jones, and Brady), umbilical view, $\times 53,134-832 \mathrm{~B}-59 \mathrm{R}-3,49-51 \mathrm{~cm} . \quad 2, \mathbf{3 , 6}$. Globorotalia (Menardella) multicamerata (Cushman and Jarvis), (2) umbilical view, $\times 61,143-832 \mathrm{~B}-70 \mathrm{R}-3,148-150 \mathrm{~cm}$, (3) umbilical view, $\times 67,143-832 \mathrm{~B}-71 \mathrm{R}-4,95-98 \mathrm{~cm}$, (6) peripheral view, $\times 80$, 143-832B-71R-4, $95-98 \mathrm{~cm}$. 4. Globorotalia (Globorotalia) tumida tumida (Brady), umbilical view, $\times 64,134-832 \mathrm{~B}-64 \mathrm{R}-5,111-113 \mathrm{~cm}$. 5. Globorotalia (Globorotalia) tumida flexuosa (Koch), peripheral view, $\times 32,134-832 \mathrm{~B}-63 \mathrm{R}-5,62 \times 64 \mathrm{~cm}$. 7. Neogloboquadrina humerosa (Takayanagi and Saito), umbilical view, $\times 107$, 134-832B-69R-1, 84-86 cm. 8. Neogloboquadrina acostaensis (Blow), umbilical view, $\times 120,134-832 \mathrm{~B}-71 \mathrm{R}-5-1,31-134 \mathrm{~cm}$. 9. Pulleniatina primalis Banner and Blow, apertural view, $\times 147,134-832 \mathrm{~B}-71 \mathrm{R}-5,131-134 \mathrm{~cm}$. 


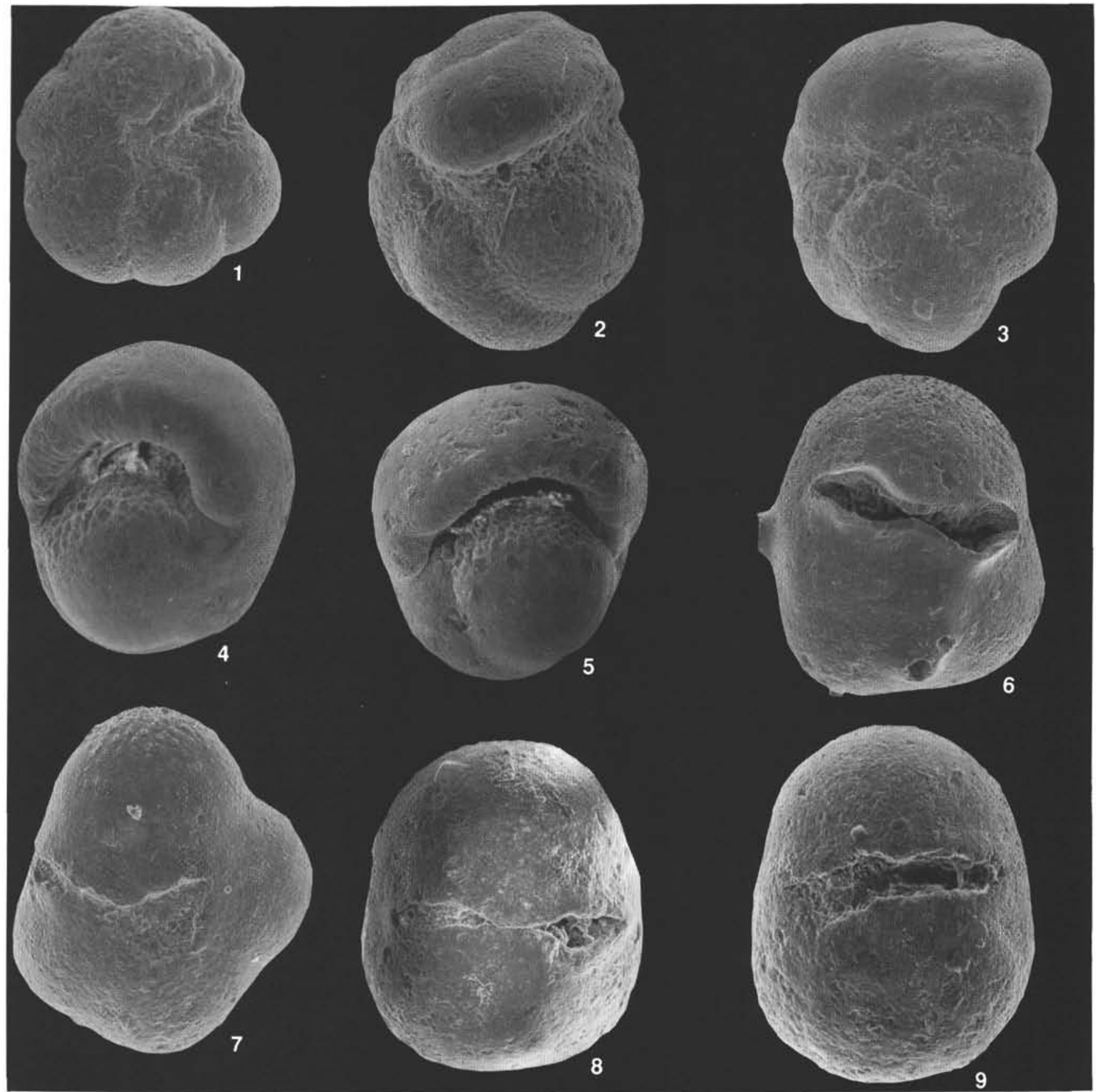

Plate 3. Biostratigraphically important species in sediments from the uppermost Miocene to upper Pliocene. 1. Neogloboquadrina humerosa, umbilical view, $\times 134,134-832 \mathrm{~B}-71 \mathrm{R}-51,31-134 \mathrm{~cm}$. 2. Pulleniatina primalis, apertural view, $\times 93,134-832 \mathrm{~B}-63 \mathrm{R}-5,62-64 \mathrm{~cm}$. 3. Pulleniatina praecursor Banner and Blow, apertural view, $\times 134,134-832 \mathrm{~B}-64 \mathrm{R}-5,111-113 \mathrm{~cm} .4$ 4, 5. Pulleniatina obliquiloculata (Parker and Jones), (4) apertural view, $\times 107$, 134-832B-59R-1, 99-101 cm, (5) apertural view, $\times 93,134-832 \mathrm{~B}-59 \mathrm{R}-1,99-101 \mathrm{~cm}$. 6. Sphaeroidinella dehiscens (Parker and Jones), lateral view, $\times 80$, 134-832B-63R-1, 124-126 cm. 7. Sphaeroidinellopsis kochi Caudri, apertural view, $\times 107,134-832 \mathrm{~B}-65 \mathrm{R}-1,47-50 \mathrm{~cm}$. 8. Sphaeroidinellopsis paenedehiscens Blow, apertural view, $\times 107,134-832 \mathrm{~B}-69 \mathrm{R}-1,84-86 \mathrm{~cm}$. 9. Sphaeroidinellopsis seminulina seminulina (Schwager), apertural view, $\times 134$, 134-832B-71R-51, 31-134 cm. 


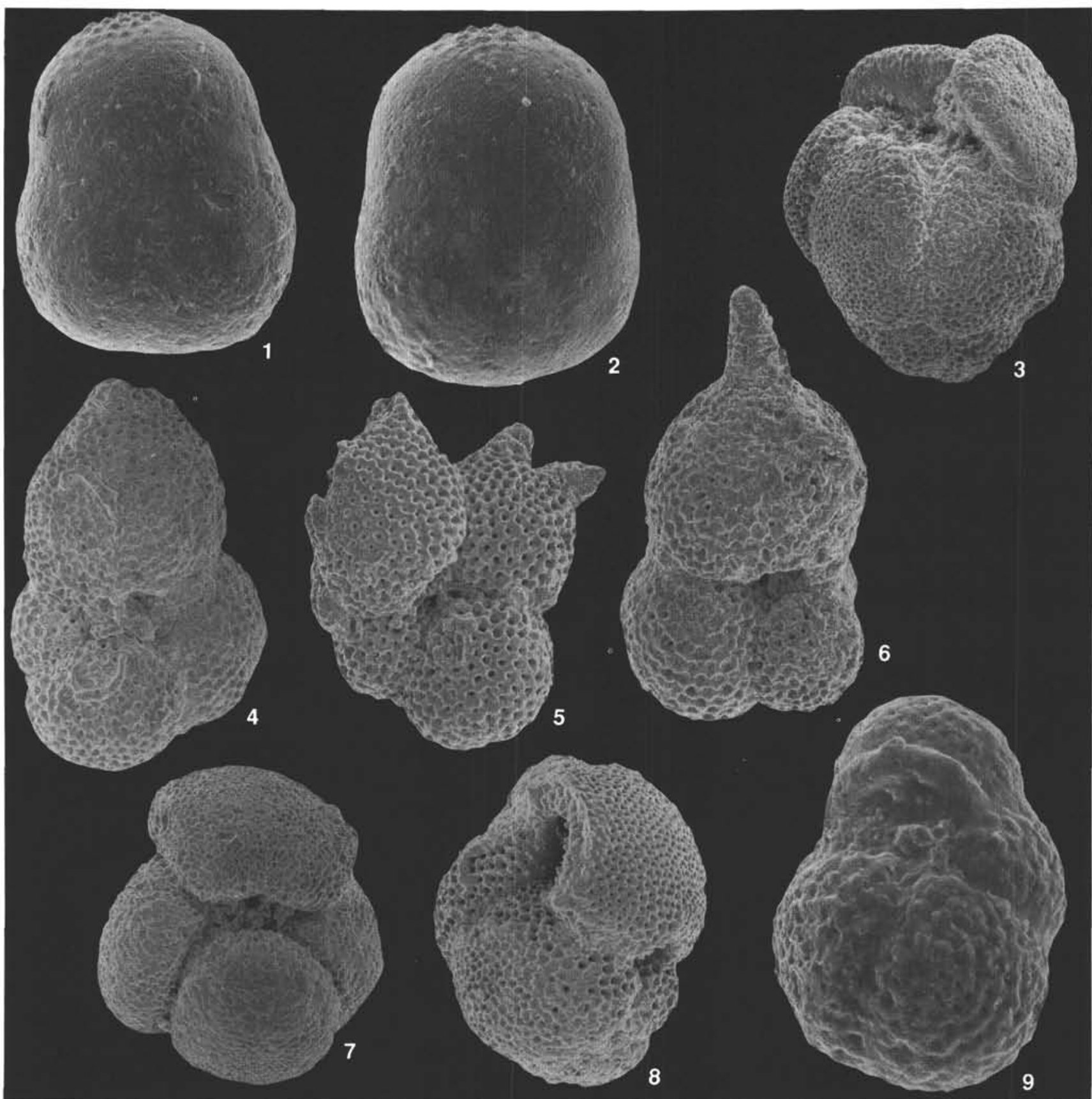

Plate 4. Biostratigraphically important species in sediments from the uppermost Miocene to upper Pliocene. 1. Sphaeroidinellopsis seminulina seminulina, spiral view, $\times 134,134-832 \mathrm{~B}-63 \mathrm{R}-3,124-126 \mathrm{~cm}$. 2. Sphaeroidinellopsis paenedehiscens, spiral view, $\times 160,134-832 \mathrm{~B}-69 \mathrm{R}-1,84-86 \mathrm{~cm} . \quad 3$. Dentoglobigerina altispira altispira (Cushman and Jarvis), lateral view, $\times 93,134-832 \mathrm{~B}-63 \mathrm{R}-5,62-64 \mathrm{~cm}$. 4. Globigerinoides sacculifer (Brady), apertural view, $\times 80,134-832 \mathrm{~B}-59 \mathrm{R}-6,49-51 \mathrm{~cm} . \quad \mathbf{5}, 6$. Globigerinoides fistulosus (Schubert), (5) apertural view, $\times 93,134-832 \mathrm{~B}-63 \mathrm{R}-3,124-126 \mathrm{~cm},(6)$ apertural view, $\times 120,134-832 \mathrm{~B}-63 \mathrm{R}-3,124-126 \mathrm{~cm}$. 7. Globigerinoides conglobatus (Brady), apertural view, $\times 67,134-832 \mathrm{~B}-63 \mathrm{R}-5,62-64 \mathrm{~cm} .8$. Globigerinoides extremus Bolli, apertural view, $\times 134,134-832 \mathrm{~B}-59 \mathrm{R}-2,99-101 \mathrm{~cm}$. 9. Globigerinoides (Zeaglobigerina) nepenthes Todd, apertural view, $\times 214,134-832 \mathrm{~B}-$ 70R-5, 145-147 cm. 


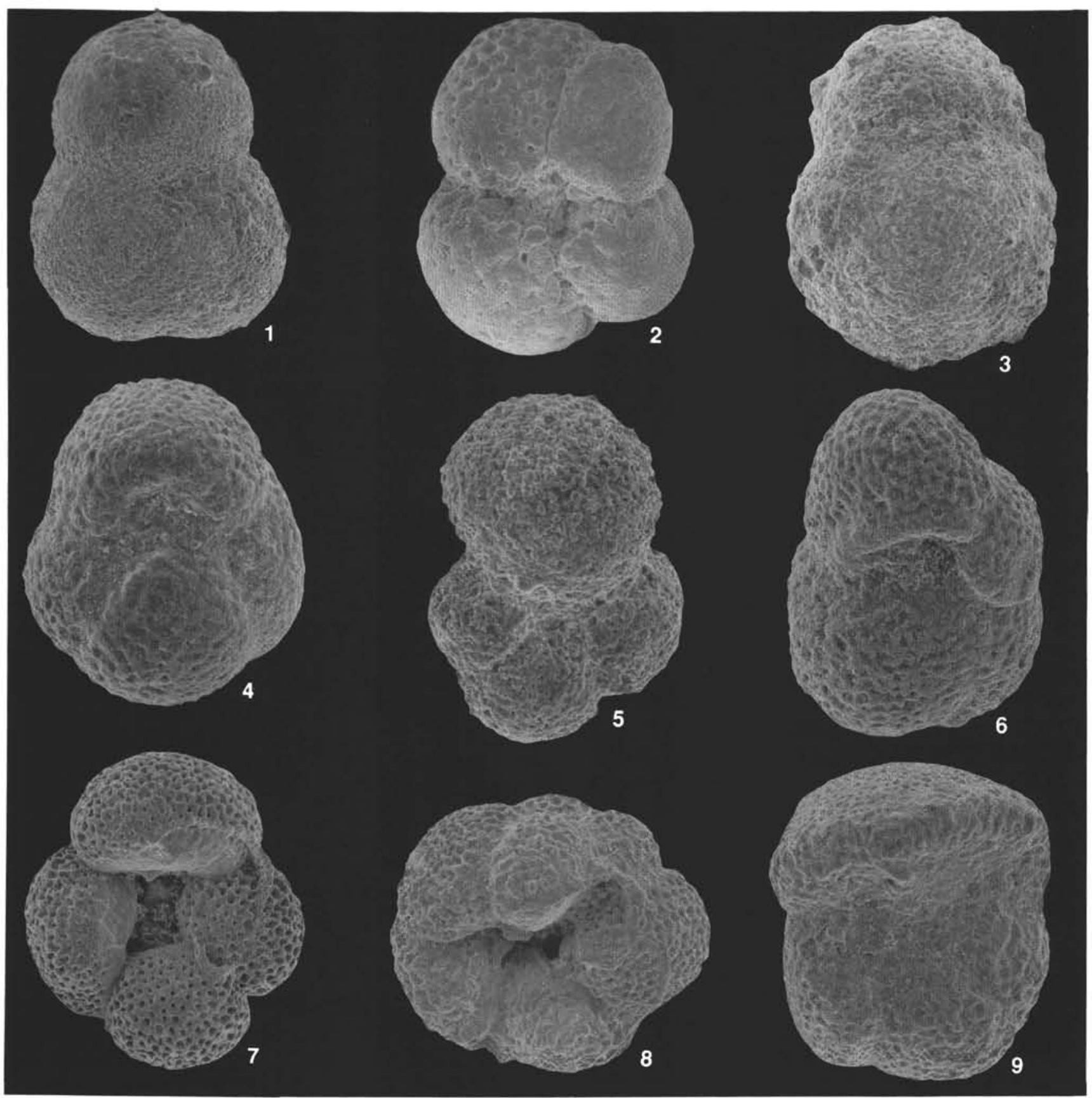

Plate 5. Biostratigraphically important species in sediments from the earliest middle Miocene to upper Miocene. 1. Globigerinoides triloba (Reuss), lateral view, $\times 160,134-832 \mathrm{~B}-90 \mathrm{R}-6,77-79 \mathrm{~cm}$. 2, 3. Globigerinoides sicanus De Stefani, (2) lateral view, $\times 147,134-832 \mathrm{~B}-90 \mathrm{R}-6,77-79 \mathrm{~cm},(3)$ lateral view, $\times 160$, 134-832B-86R-3-CC. 4. Orbulina suturalis Brönnimann, lateral view, $\times 93,134-832 B-85 R-2,29-32 \mathrm{~cm}$. 5. Orbulina universa d'Orbigny, lateral view, $\times 80,134-832 \mathrm{~B}-85 \mathrm{R}-2,29-32 \mathrm{~cm}$. 6. Praeorbulina transitoria (Blow), lateral view, $\times 134,134-832 \mathrm{~B}-90 \mathrm{R}-6,77-79 \mathrm{~cm}$. 7. Globigerinoides diminutus Bolli, apertural view, $\times 187,134-832 \mathrm{~B}-90 \mathrm{R}-6,77-79 \mathrm{~cm}$. 8. Globigerinoides subquadratus Brönnimann, apertural view, $\times 187,134-832 \mathrm{~B}-90 \mathrm{R}-6$, $77-79 \mathrm{~cm}$. 9. Globigerina (Zeaglobigerina) decoraptera Takayanagi and Saito, $\times 134,134-832 \mathrm{~B}-85 \mathrm{R}-2,29-32 \mathrm{~cm}$. 


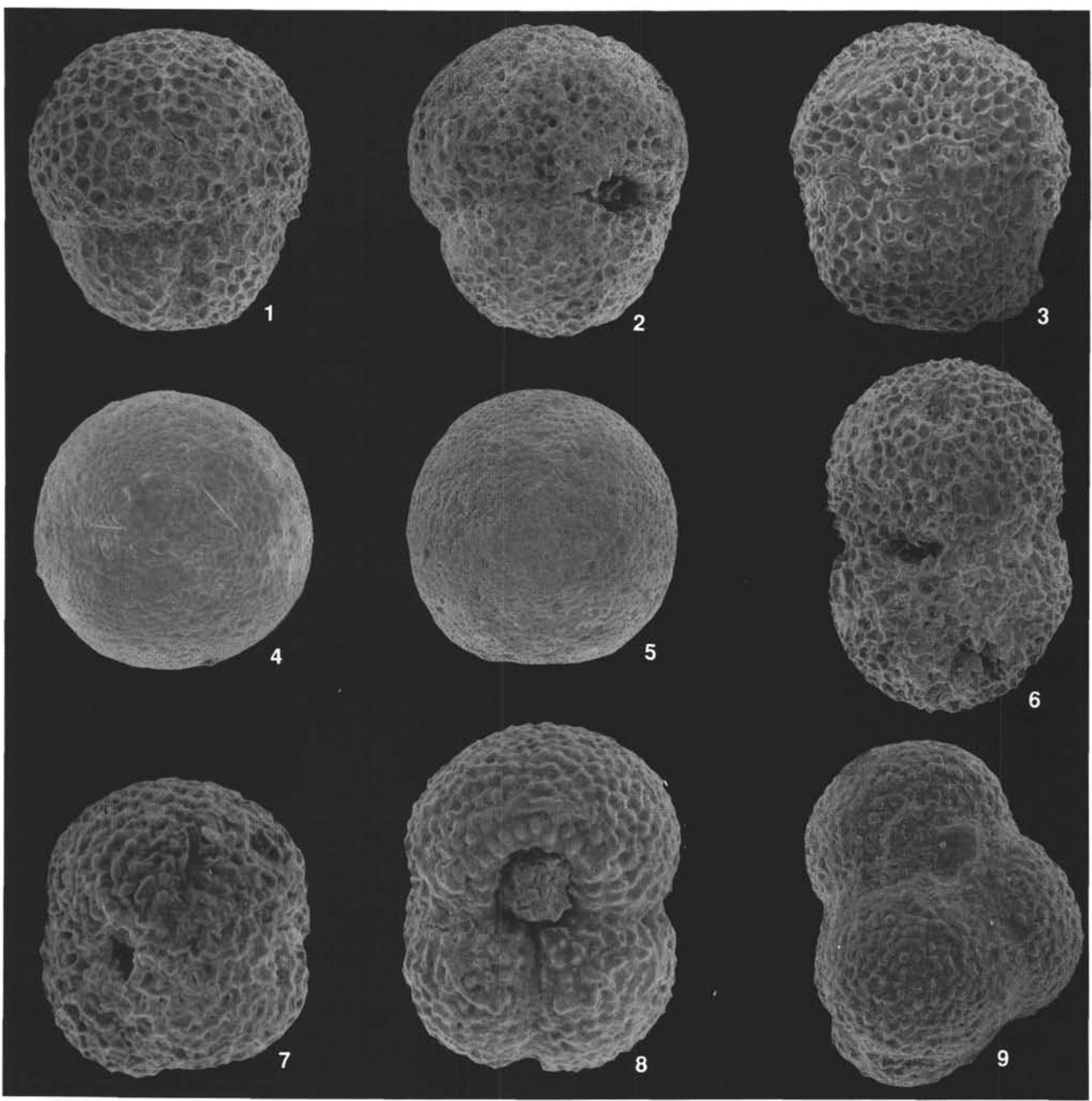

Plate 6. Biostratigraphically important species in sediments from the earliest middle Miocene to upper Miocene. 1. Sphaeroidinellopsis seminulina seminulina, apertural view, $\times 134,134-832 \mathrm{~B}-85 \mathrm{R}-3,7-11 \mathrm{~cm}$. 2. Sphaeroidinellopsis kochi Caudri, apertural view, $\times 107,134-832 \mathrm{~B}-85 \mathrm{R}-2,82-84 \mathrm{~cm} . \quad 3$. Orbulina bilobata (d'Orbigny), lateral view, $\times 107,134-832 \mathrm{~B}-74 \mathrm{R}-2,123-124 \mathrm{~cm}$. 4. Globigerina (Zeaglobigerina) druryi Akers, apertural view, $\times 174$, 134-832B-85R-2, 29-32 cm. 5. Globigerinella praesiphonifera (Blow), umbilical view, $\times 174,134-832 \mathrm{~B}-90 \mathrm{R}-6,77-79 \mathrm{~cm}$. 6. Globigerina (Zeaglobigerina) nepenthes, umbilical view, $\times 160,134-832 \mathrm{~B}-85 \mathrm{R}-3,7-11 \mathrm{~cm}$. 7. Globoquadrina baroemoenensis (LeRoy), apertural view, $\times 120,134-832 \mathrm{~B}-85 \mathrm{R}-2$, $82-84 \mathrm{~cm}$. 8. Dentoglobigerina altispira altispira, apertural view, $\times 120,134-832 \mathrm{~B}-85 \mathrm{R}-2,82-84 \mathrm{~cm}$. 9. Globoquadrina dehiscens (Chapman, Parr, and Collins), apertural view, $\times 134,134-832 \mathrm{~B}-85 \mathrm{R}-2,29-32 \mathrm{~cm}$. 